DESY 12-112

June 2012

\title{
Decaying vs Annihilating Dark Matter in Light of a Tentative Gamma-Ray Line
}

\author{
Wilfried Buchmüller and Mathias Garny \\ Deutsches Elektronen-Synchrotron DESY, 22607 Hamburg, Germany
}

\begin{abstract}
Recently reported tentative evidence for a gamma-ray line in the Fermi-LAT data is of great potential interest for identifying the nature of dark matter. We compare the implications for decaying and annihilating dark matter taking the constraints from continuum gamma-rays, antiproton flux and morphology of the excess into account. We find that higgsino and wino dark matter are excluded, also for nonthermal production. Generically, the continuum gamma-ray flux severely constrains annihilating dark matter. Consistency of decaying dark matter with the spatial distribution of the Fermi-LAT excess would require an enhancement of the dark matter density near the Galactic center.
\end{abstract}




\section{Introduction}

Monochromatic gamma-ray lines have been suggested long ago as signature for pairannihilation of dark matter (DM) particles [1]. During the past decades many DM candidates have been discussed, the most popular ones being weakly interacting massive particles (WIMPs) in the context of supersymmetric extensions of the Standard Model [2]. More recently, also decaying dark matter has been studied in detail [3 6]. An attractive feature of decaying gravitino dark matter is the consistency with thermal leptogenesis, contrary to standard WIMP dark matter [4].

During the past years the Large Area Telescope (LAT) [7], on board the Fermi gamma-ray space telescope, has searched with unprecedented sensitivity for photon lines from $30 \mathrm{MeV}$ to $300 \mathrm{GeV}$. Stringent constraints on decaying and annihilating dark matter have been obtained from searches in the energy range $30-200 \mathrm{GeV}$ based on 11 months of data [8], and in the range $7-200 \mathrm{GeV}$ based on 23 months of data 9 . The search region used in these analyses covers the whole sky except for the Galactic disk $\left(|b|>10^{\circ}\right)$ plus a $20^{\circ} \times 20^{\circ}$ region around the Galactic center. A similar analysis has been performed independently based on publicly available data corresponding to 27 months in Ref. [10]. No indications for gamma-ray lines were found.

In a recent analysis, that is based on optimized search regions around the Galactic center, and takes 43 months of Fermi-LAT data into account, a hint for a gamma-ray feature in the energy range $120-140 \mathrm{GeV}$ is reported [11,12]. When interpreted in terms of a gamma-ray line [12], the significance obtained from the statistical uncertainties in the search regions close to the Galactic center is $4.6 \sigma$. The significance is reduced to $3.3 \sigma$ when correcting for the bias introduced by selecting the search regions. While the excess is currently under an active debate [13 18], the claim has been further strengthened by a recent analysis [19], which confirms the existence of an excess, as well as its spectral shape, with even higher statistical significance than the one claimed in Ref. [12]. In addition, indications are found that the excess originates from a relatively narrow region of a few degrees around the Galactic center, possibly with a small offset within the Galactic plane. Hopefully, the question about the existence of the spectral feature and its precise properties will be settled in the near future.

Assuming that the feature is real, the question about its origin is of great interest. While an astrophysical explanation might eventually be identified, a spectral feature in this energy range can arise rather generically from the annihilation or decay of dark 
matter particles. In this paper we compare decaying and annihilating dark matter based on two prototype models: decaying gravitinos and wino/higgsino-like WIMPs which annihilate predominantly into two W-bosons. In both cases we treat the branching ratios into $\gamma \nu$ (gravitino) and $\gamma \gamma$ (wino/higgsino) final states as free parameters to account for some model dependence. Note that we do not demand thermal freeze-out for WIMPs. Higgsinos and winos can be nonthermally produced in gravitino decays, compatible with leptogenesis [20,21], or, alternatively, in moduli decays [22 24].

In the following we shall compare interpretations of the tentative $130 \mathrm{GeV}$ photon line in terms of decaying and annihilating dark matter in a sequence of increasing assumptions: We first consider constraints from continuum gamma-rays, which are independent of charged cosmic rays and the dark matter distribution (Section 2). This is followed by a discussion of constraints from antiprotons, which depend on the propagation model (Section 3). We then analyze the implications of the spatial distribution of the Fermi-LAT excess (Section 4) and draw our conclusions (Section 5).

\section{Constraints from continuum gamma-rays}

Since the dark matter particle is required to be electrically neutral, the annihilation or decay into photons is typically suppressed compared to channels involving for example electroweak gauge bosons or the Higgs boson, as well as quarks or leptons. The subsequent decay and fragmentation of these annihilation or decay products gives rise, among others, to an emission of gamma rays with a broad spectrum in the energy range $\simeq 1-100 \mathrm{GeV}$ below the line. Therefore, one expects that the line flux is accompanied by an associated continuum flux with the same spatial distribution, whose strength depends on the relative size of the annihilation or decay rates into photons and into other Standard Model particles. As compared to the sharp feature resulting from the direct decay into photons, the continuum emission has a less characteristic spectral shape and is therefore much harder to disentangle from the background gamma-ray flux in the Galactic center region [25]. On the other hand, one expects a much larger total flux in the continuum compared to the line, and therefore it is important to check up to which level a continuum emission is acceptable. Apart from the center of the Galaxy, the continuum flux of gamma rays can be constrained, e.g., by the emission seen within the Galactic halo [26], from Galaxy clusters [27, 28], or from dwarf galaxies [29]. In general, a comparison of the various constraints from different targets is 
affected by the uncertainties related to the dark matter density distribution in the different environments.

We will not enter into the details of this discussion here, but instead follow a very conservative approach. Namely, we consider the same data sets for the photon flux that have been used in Ref. [12 to search for a gamma-ray line signal. However, instead of using only a line as the template, we add a contribution from the continuum flux, and test whether the combined spectrum yields a satisfactory fit to the data. This strategy has the advantage that it is not affected by uncertainties related to the actual dark matter distribution, because both the line and the continuum fluxes are generated from the same source. Concretely, we consider a spectral template for the fit given by

$$
\frac{d J}{d E}=\alpha\left(\delta\left(E-E_{\gamma}\right)+\frac{d N_{E G}}{d E}+\frac{1-\mathrm{BR}_{\gamma}}{N_{\gamma} \mathrm{BR}_{\gamma}} \frac{d N_{c o n t}^{\gamma}}{d E}\right)+\beta\left(\frac{E}{E_{\gamma}}\right)^{-\gamma},
$$

where $E_{\gamma}=m_{D M}\left(m_{D M} / 2\right), \mathrm{BR}_{\gamma}=\sigma v_{\gamma \gamma} / \sigma v_{t o t}\left(\tau / \tau_{\gamma \nu}\right)$ and $N_{\gamma}=2(1)$ for dark matter annihilation (decay). The fit parameter $\alpha$ determines the strength of the dark matter flux, while $\beta$ and $\gamma$ model the contribution from backgrounds, assumed to follow a power law within the considered energy range (see [13] for possible caveats). For a given dark matter profile and search region, $\alpha$ can be easily converted into the dark matter annihilation cross section $\sigma v_{\gamma \gamma}$ or partial lifetime $\tau_{\gamma \nu}$ into monochromatic photons. The continuum spectrum is in general given by the sum of two components. The first one arises from redshifted monochromatic photons with extragalactic origin. For decaying dark matter it is given by 30

$$
\frac{d N_{E G}}{d E}=\frac{\Omega_{D M} \rho_{c 0}}{\sqrt{\Omega_{M}}\left(H_{0} / c\right) \bar{J}_{\psi}} \frac{E^{1 / 2}}{E_{\gamma}^{3 / 2}}\left(1+\frac{\Omega_{\Lambda}}{\Omega_{M}}\left(\frac{E}{E_{\gamma}}\right)^{3}\right)^{-1 / 2} \Theta\left(E_{\gamma}-E\right) .
$$

Here $\Omega_{\Lambda}=1-\Omega_{M} \simeq 0.73$ are the cosmological density parameters, assuming a spatially flat $\Lambda$ CDM model, $\Omega_{D M} h^{2}=0.112$ is the dark matter density, $H_{0}=h 100 \mathrm{~km} / \mathrm{s} / \mathrm{Mpc}$ is the Hubble constant with $h=0.704, \rho_{c 0}=1.05 \cdot 10^{-5} h^{2} \mathrm{GeV} / \mathrm{cm}^{3}$ is the critical density, $c$ is the speed of light, and the factor $\bar{J}_{\psi}=\Delta \Omega^{-1} \int_{\Delta \Omega} d \Omega \int_{\text {l.o.s. }} \rho_{d m}(r)$ appears because of the normalization chosen in (2.1). For the search regions 3 and 4 of [12] and the various density profiles, the prefactor in 2.2 is of the order $0.3-0.4$.

The second contribution to the continuum gamma spectrum arises from the weighted sum of the spectra produced by annihilation or decays, excluding $\gamma \gamma(\gamma \nu)$,

$$
\frac{d N_{c o n t}^{\gamma}}{d E} \equiv \frac{1}{\sum_{f \neq \gamma} \mathrm{BR}_{f}} \sum_{f \neq \gamma} \mathrm{BR}_{f} \frac{d N_{f}^{\gamma}}{d E}
$$


where $d N_{f}^{\gamma} / d E$ is the number of photons per energy and per annihilation/decay resulting from each mode, and $\mathrm{BR}_{f}$ is the corresponding branching ratio. For the line, we use the same shape as in [12] based on the instrument response function of the Fermi-LAT detector $(\mathrm{FWHM}=0.136)$, which has somewhat broader tails compared to a Gaussian, and also smooth the step function appearing in the extragalactic contribution (2.2) accordingly. The continuum flux is determined using PYTHIA 8.1 [31]. In order to increase the sensitivity to the continuum flux we use the data presented in [12] over the full energy range $20-300 \mathrm{GeV}$, taking the more finely binned data where they are available $(80-200 \mathrm{GeV})$. Lastly, we require that $\gamma>2.0$ as a conservative lower limit for the background power law index [32], while its normalization is freely varied. For performing the fit we use the profile likelihood method as detailed in [12], with 95\% C.L. statistical errors obtained by varying $\alpha$ (with fixed $\mathrm{BR}_{\gamma}$ ) and profiling over $\beta$ and $\gamma$ until the TS-value has decreased by 3.84. We checked that our statistical analysis reproduces the best-fit values and confidence intervals in [12] using the smaller energy window and a single line up to $10 \%$ for regions $2-4$, both data samples and all density profiles. When including $\mathrm{BR}_{\gamma}$ in the fit, we use $\Delta(T S)=5.99$ to obtain the $95 \%$ C.L. regions, corresponding to the $\chi_{k=2}^{2}$ distribution that is expected for two parameters of interest ${ }^{1}$. We will comment on the dependence on the various assumptions later on.

\subsection{Decaying dark matter}

For decaying gravitino dark matter, which we consider as representative example, we take the decay channels $Z \nu, W \ell, h \nu$ apart from $\gamma \nu$ into account. In the appendix, the partial decay widths are given in the case of bilinear R-parity breaking as functions of the gravitino mass, parameters of R-parity breaking, gaugino masses and the higgsino mass parameter $\mu$. According to Eq. (5.19), the branching ratio into $\gamma \nu$ is enhanced for hierarchical gaugino masses. For example, in the case of wino NLSP with $M_{2} \simeq m_{3 / 2}$ one obtains the maximal branching ratio,

$$
\mathrm{BR}_{\gamma}^{\max } \simeq \frac{3 \pi \alpha}{2 \sqrt{2} G_{F} m_{3 / 2}^{2}},
$$

\footnotetext{
${ }^{1}$ By generating a large sample of data that would be expected in the presence of a true signal, we checked that the actual distribution of the profile likelihood ratio is well described by $\chi_{k=1}^{2}$ when keeping $\mathrm{BR}_{\gamma}$ fixed, while it is somewhat steeper than $\chi_{k=2}^{2}$ when including $\mathrm{BR}_{\gamma}$ in the fit, lying between a $\chi_{k=1}^{2}$ and $\chi_{k=2}^{2}$ distribution. Therefore the confidence regions assuming $\chi_{k=2}^{2}$ may be regarded as conservative.
} 
which yields $\mathrm{BR}_{\gamma}^{\max } \simeq 3 \%$ for $m_{3 / 2} \simeq 260 \mathrm{GeV}$. On the contrary, for higgsino NLSP, with $\mu \simeq m_{3 / 2} \ll M_{1}, M_{2}$, the branching ratio into $\gamma \nu$ is negligible.

\begin{tabular}{|c|c|c|c|c|c|c|c|c|c|c|c|c|}
\hline \multicolumn{2}{|c|}{ Reg } & $\begin{array}{c}E_{\gamma} \\
{[\mathrm{GeV}]}\end{array}$ & $\gamma$-ray lin & \multicolumn{8}{|c|}{$\tau_{\gamma \nu}\left[10^{28} \mathrm{~s}\right]$} & $=0.001$ \\
\hline 3 & $\mathrm{~S}$ & 128.1 & $1.49_{-0.50}^{+1.30}$ & $4.5 \sigma$ & $1.46_{-0.50}^{+1.27}$ & $4.5 \sigma$ & $1.28_{-0.41}^{+1.04}$ & $4.6 \sigma$ & $3.42_{-0.84}^{+3.26}$ & $3.2 \sigma$ & 11.7 & $1.0 \sigma$ \\
\hline 3 & $\mathrm{UC}$ & 129.5 & $1.94_{-0.74}^{+2.30}$ & $3.8 \sigma$ & $1.88_{-0.71}^{+2.23}$ & $3.8 \sigma$ & $1.60_{-0.58}^{+1.73}$ & $4.0 \sigma$ & $3.27_{-0.71}^{+3.72}$ & $3.1 \sigma$ & 11.4 & $0.9 \sigma$ \\
\hline 4 & $\mathrm{~S}$ & 129.8 & $1.24_{-0.44}^{+1.20}$ & $4.3 \sigma$ & $1.21_{-0.43}^{+1.16}$ & $4.3 \sigma$ & $1.10_{-0.35}^{+0.95}$ & $4.5 \sigma$ & $3.54_{-1.02}^{+7.86}$ & $2.6 \sigma$ & 21.1 & $0.6 \sigma$ \\
\hline 4 & $\mathrm{UC}$ & 129.9 & $1.66_{-0.67}^{+2.41}$ & $3.5 \sigma$ & $1.62_{-0.65}^{+2.30}$ & $3.5 \sigma$ & $1.38_{-0.51}^{+1.70}$ & $3.7 \sigma$ & $3.67_{-1.07}^{+11.9}$ & $2.4 \sigma$ & 21.7 & $0.6 \sigma$ \\
\hline
\end{tabular}

Table 1: Partial dark matter lifetime $\tau_{\gamma \nu}$ in the monochromatic gamma channel obtained from a fit to the Fermi-LAT data in the energy range $20-300 \mathrm{GeV}$ in search regions 3 and 4 of Ref. 12, for the SOURCE (S) and ULTRACLEAN (UC) data samples, for various fixed values of the branching ratio $\mathrm{BR}_{\gamma \nu}=\tau / \tau_{\gamma \nu}$ and using the Einasto profile. The columns show the line energy that yields the best fit, the lifetimes with 95\%C.L. statistical errors, and the significance $\sigma \equiv \sqrt{T S}$ (without trial corrections) with respect to the background-only case.

In order to illustrate the possibilities to explain the tentative gamma-ray line with decaying dark matter, we show in Fig. 1 the photon spectrum from gravitino decay, fitted to the flux measured by Fermi LAT within the search region 3 of Ref. [12], for three values of the branching ratio into monochromatic photons. The feature in the spectrum near $130 \mathrm{GeV}$ can be explained for branching ratios of $10 \%$ and $1 \%$. For the relatively small value $0.25 \%$, the continuum gamma-ray contribution becomes so strong that it would overshoot the measured flux if one would require the monochromatic decay mode to account for the feature in the spectrum. In this case, the fit to the observed spectrum becomes worse, and requires a somewhat longer total lifetime, such that the continuum flux is in accordance with the observed one. The best fit values for the partial lifetime, as well as the significance with respect to the background-only case obtained from search regions 3 and 4 are shown in Tab. 1, for various branching ratios. The significance of the fit remains nearly constant for branching ratios larger than $1 \%$, and then steeply decreases. For a branching ratio of $0.1 \%$ or smaller, the dark matter component cannot yield a significantly better description than the background only.

We have also performed a fit where the branching ratio is varied together with the parameters $\alpha, \beta, \gamma$ in Eq. (2.1). The resulting 95\%C.L. contours are shown in Fig. 2, indicating again that branching ratios below $0.25 \%$ are disfavored because of the continuum contribution to the photon flux. For comparison we also show the 
Decaying Dark Matter (SOURCE)

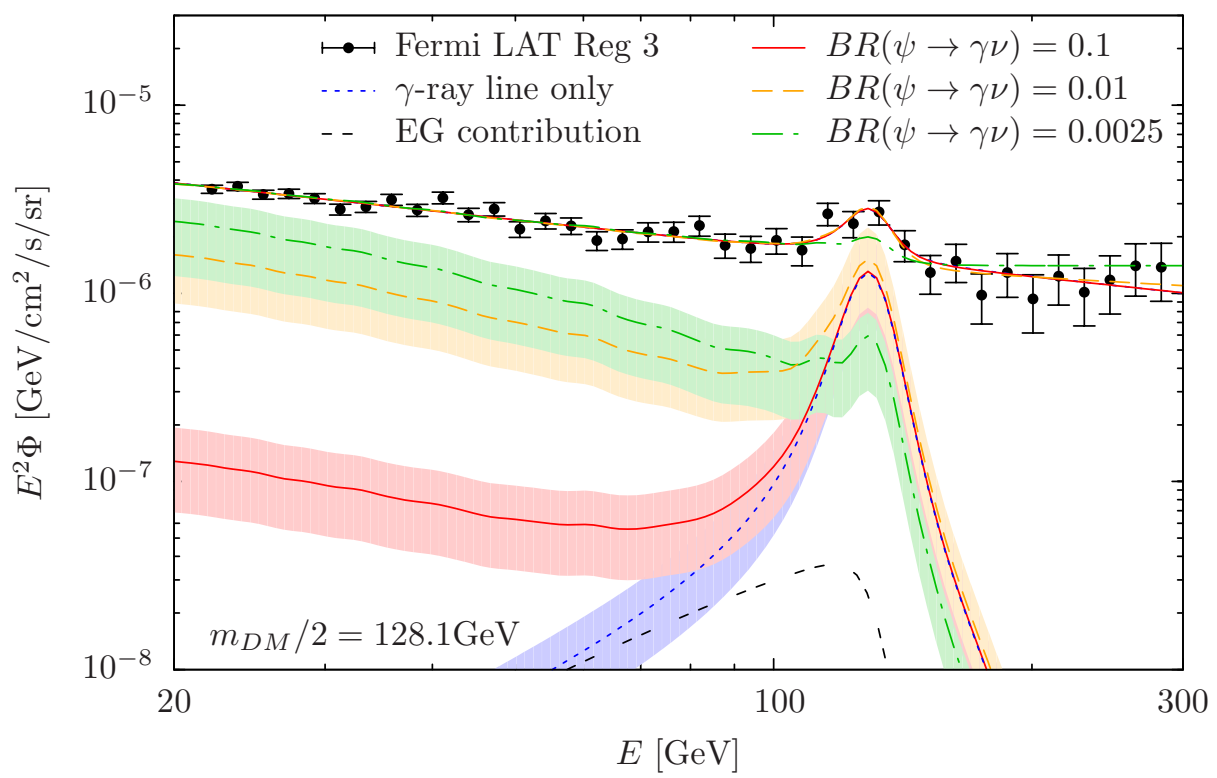

Decaying Dark Matter (ULTRACLEAN)

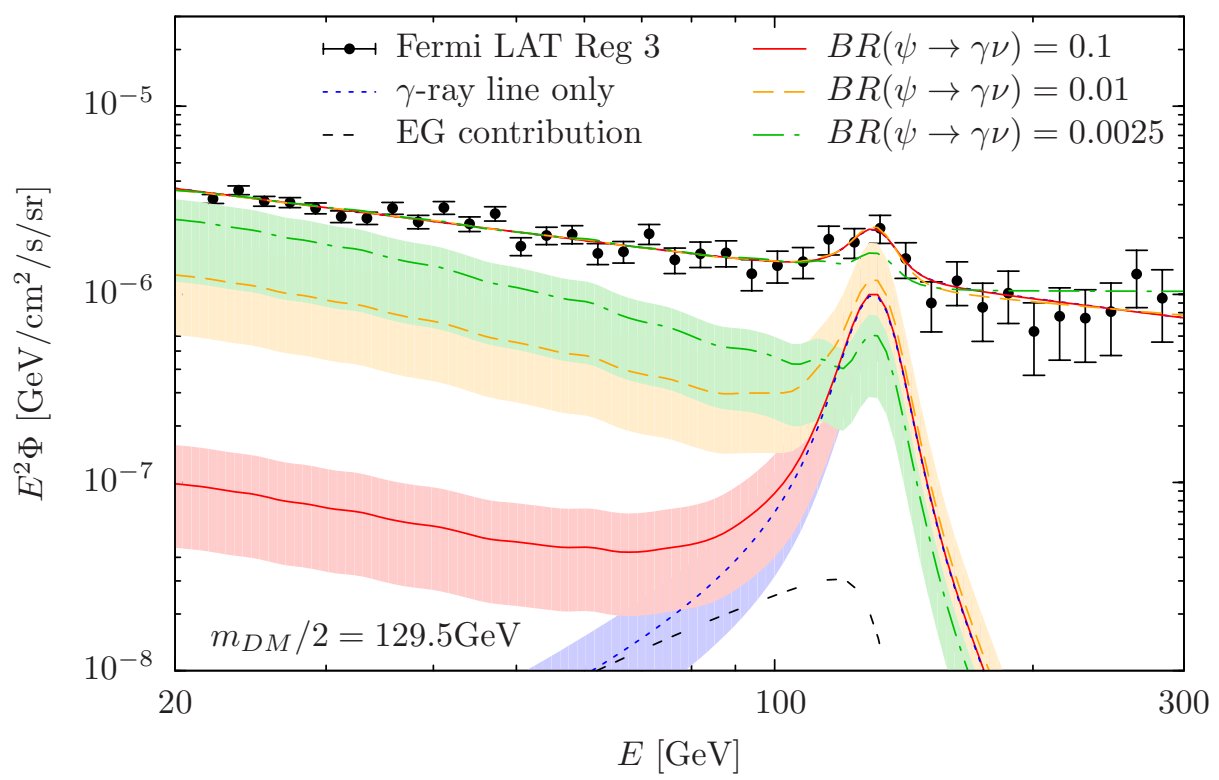

Figure 1: Sum of monochromatic and continuum photon flux originating from dark matter decaying into $\gamma \nu, Z \nu, h \nu, W^{ \pm} \ell^{\mp}$ with branching ratios $\mathrm{BR}_{\gamma}=0.1,0.01$, and 0.0025 , respectively. The lower lines show the contribution from dark matter decay, and the upper lines the sum of signal and fitted power-law background. The dashed blue line shows the flux when taking the gamma-ray line only, plus the redshifted extragalactic contribution, into account. The gamma-ray flux measured by Fermi-LAT corresponding to the SOURCE (upper figure) and ULTRACLEAN (lower figure) data samples is taken from Ref. 12. The shaded regions correspond to 95\%C.L. error bands. 

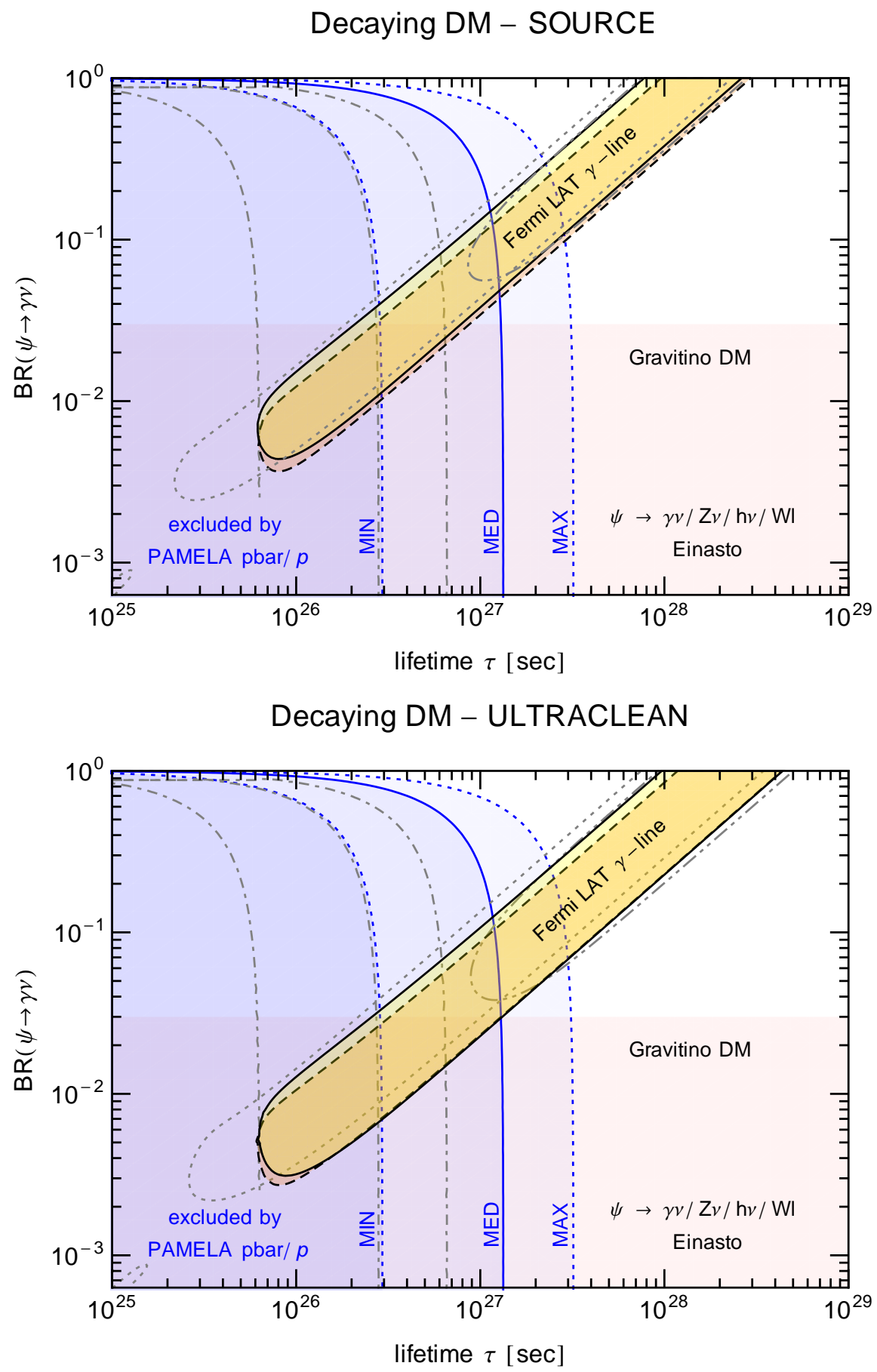

Figure 2: Combined fit of the monochromatic line at $\approx 130 \mathrm{GeV}$ as well as the continuum gamma-ray spectrum from dark matter decay. The yellow and orange shaded areas are 95\%C.L. regions for the total dark matter lifetime $\tau$ and the branching ratio $\operatorname{BR}(\psi \rightarrow \gamma \nu)$ obtained from the Fermi-LAT data within search regions 3 and 4 or Ref. [12, respectively. The regions excluded from antiproton constraints at 95\%C.L. based on the PAMELA $\bar{p} / p$ data are shown as blue shaded regions, as well as the expected range for gravitino dark matter (red shaded area). Further explanations are given in the text. 
more stringent constraints resulting from fixing the background parameters $(\beta, \gamma)=$ $\left.(\beta, \gamma)\right|_{B R_{\gamma \nu}=1}$ to the best-fit values obtained from the fit using a gamma-ray line only (grey dot-dashed line), as well as the constraints obtained when using the smaller energy range $80-200 \mathrm{GeV}$ (grey dotted line) for region 4. We also note that the results are rather insensitive to the precise choice of the relative branching ratios among $Z \nu, W \ell, h \nu$.

Clearly, the limits on the continuum gamma-ray flux obtained in this way are very conservative ones, while stronger constraints could be obtained, e.g., by taking photons produced via inverse Compton scattering into account (e.g. [5]) or extending the energy range below $20 \mathrm{GeV}$. However, this would also make the conclusions more dependent on details of the background spectrum and therefore on the astrophysical processes close to the Galactic center. In this sense, the limits presented here may be regarded as a rather robust consistency check, based on the assumption that the background can be modeled by a power law in the energy range $20-300 \mathrm{GeV}$.

\subsection{Annihilating dark matter}

One of the most studied candidates for dark matter is the lightest neutralino within the MSSM, for which the annihilation cross sections into $\gamma \gamma$ and $\gamma Z$, that yield monochromatic gamma rays, are loop suppressed [33]. Consequently, the branching ratios into these final states are typically at the permille level, and the continuum flux dominates the gamma spectrum.

In the following we perform first a model independent analysis in order to determine the requirements on the branching ratio into monochromatic photons, and then check whether some well-motivated scenarios are compatible with these constraints.

In particular, we first assume that dark matter annihilates into $\gamma \gamma$ and $W^{+} W^{-}$ final states, giving rise to a line at $E_{\gamma}=m_{D M}$ and a continuum photon flux in the energy range $E<m_{D M}$. The resulting photon flux is shown in Fig. 3 , for several values of the branching ratio $\mathrm{BR}_{\gamma}=\sigma v_{\gamma \gamma} /\left(\sigma v_{\gamma \gamma}+\sigma v_{W W}\right)$. Similarly to decaying dark matter, we find that the feature in the Fermi spectrum at $\approx 130 \mathrm{GeV}$ can be well fitted for branching ratios $10 \%$ or $1 \%$, while the continuum spectrum becomes dominant for $0.25 \%$. Note that the photon spectrum exhibits a more pronounced dip between the continuum and monochromatic contributions as compared to decaying dark matter, due to the absence of the extragalactic contribution. The statistical significance and 


\begin{tabular}{|c|c|c|c|c|c|c|c|c|c|c|c|c|}
\hline \multirow{2}{*}{\multicolumn{2}{|c|}{ Reg }} & \multirow{3}{*}{$\begin{array}{c}\begin{array}{c}E_{\gamma} \\
{[\mathrm{GeV}]}\end{array} \\
127.9\end{array}$} & \multicolumn{10}{|c|}{$\sigma v_{\gamma \gamma}\left[10^{-27} \mathrm{~cm}^{3} / \mathrm{s}\right]$} \\
\hline & & & \multicolumn{2}{|c|}{$\gamma$-ray line only } & \multicolumn{2}{|c|}{$\mathrm{BR}_{\gamma \gamma}=0.1$} & \multicolumn{2}{|c|}{$\mathrm{BR}_{\gamma \gamma}=0.01$} & \multicolumn{2}{|c|}{$\mathrm{BR}_{\gamma \gamma}=0.0025$} & \multicolumn{2}{|c|}{$\mathrm{BR}_{\gamma \gamma}=0.001$} \\
\hline 3 & $\mathrm{~S}$ & & $1.25_{-0.58}^{+0.65}$ & $4.5 \sigma$ & $1.24_{-0.58}^{+0.64}$ & $4.5 \sigma$ & $0.99_{-0.49}^{+0.52}$ & $4.1 \sigma$ & $0.32_{-0.23}^{+0.21}$ & $2.7 \sigma$ & 0.10 & $1.7 \sigma$ \\
\hline 3 & $\mathrm{UC}$ & 129.3 & $0.98_{-0.54}^{+0.64}$ & $3.8 \sigma$ & $0.97_{-0.53}^{+0.60}$ & $3.8 \sigma$ & $0.80_{-0.47}^{+0.51}$ & $3.5 \sigma$ & $0.29_{-0.25}^{+0.22}$ & $2.3 \sigma$ & 0.09 & $1.3 \sigma$ \\
\hline 4 & $\mathrm{~S}$ & 129.6 & $1.24_{-0.62}^{+0.68}$ & $4.3 \sigma$ & $1.21_{-0.61}^{+0.68}$ & $4.2 \sigma$ & $0.86_{-0.48}^{+0.51}$ & $3.6 \sigma$ & 0.22 & $1.9 \sigma$ & 0.05 & $0.8 \sigma$ \\
\hline 4 & $\mathrm{UC}$ & 129.8 & $0.93_{-0.55}^{+0.63}$ & $3.5 \sigma$ & $0.92_{-0.55}^{+0.63}$ & $3.5 \sigma$ & $0.68_{-0.46}^{+0.50}$ & $3.0 \sigma$ & 0.19 & $1.6 \sigma$ & 0.04 & $0.6 \sigma$ \\
\hline
\end{tabular}

Table 2: Annihilation cross section in the monochromatic gamma channel obtained from a fit to the Fermi-LAT data in the energy range $20-300 \mathrm{GeV}$ in search regions 3 and 4 of Ref. 12, for the SOURCE (S) and ULTRACLEAN (UC) data samples, for various fixed values of the branching ratio $\mathrm{BR}_{\gamma \gamma}=\sigma v_{\gamma \gamma} /\left(\sigma v_{\gamma \gamma}+\sigma v_{W W}\right)$, and using the Einasto profile. The columns show the line energy that yields the best fit, the cross sections with 95\%C.L. statistical errors, and the significance $\sigma \equiv \sqrt{T S}$ (without trial corrections) with respect to the background-only case. The small differences in the statistical significances between dark matter decay (see Tab. 1) and annihilation are mainly due to the extragalactic contribution as well as a slightly different continuum spectrum.

best-fit values of the cross section are given in Tab. 2 for various branching ratios, and exhibit a similar dependence than for decaying dark matter. One might notice that the significance of the fit always decreases when lowering $\mathrm{BR}_{\gamma}$, while for decaying dark matter it is slightly better for $\mathrm{BR}_{\gamma} \sim 1 \%$. However, these differences are too small to be taken seriously. A common feature is the drastic decrease in the significance for branching ratios smaller than $1 \%$. As before, we also performed a fit with variable branching ratio. The resulting 95\%C.L. regions are shown in Fig. 4. Depending on the search region and on the data sample used for the analysis, we find that a branching ratio larger than $0.4-0.8 \%$ is required. For comparison, the constraints obtained when using a fixed background contribution (grey dot-dashed line) or when using the smaller energy range $80-200 \mathrm{GeV}$ (grey dotted lines) are shown as well. The sensitivity to the continuum spectrum is essentially lost in the latter case for annihilating dark matter, due to the drop of the flux below the line. On the other hand, we checked that removing the lower limit $\gamma>2.0$ on the background slope does not affect the confidence regions.

As a second generic example, we consider dark matter annihilating into $\gamma Z$ and $\gamma \gamma$, giving rise to two lines at $E_{\gamma, 1}=m_{D M}$ and $E_{\gamma, 2}=m_{D M}-M_{Z}^{2} /\left(4 m_{D M}\right)$. In addition, annihilations into $W^{+} W^{-}$and $Z Z$ yield a continuum spectrum. Motivated by higgsino dark matter, we fix the relative contribution of the two lines according 
Annihilating Dark Matter (SOURCE)

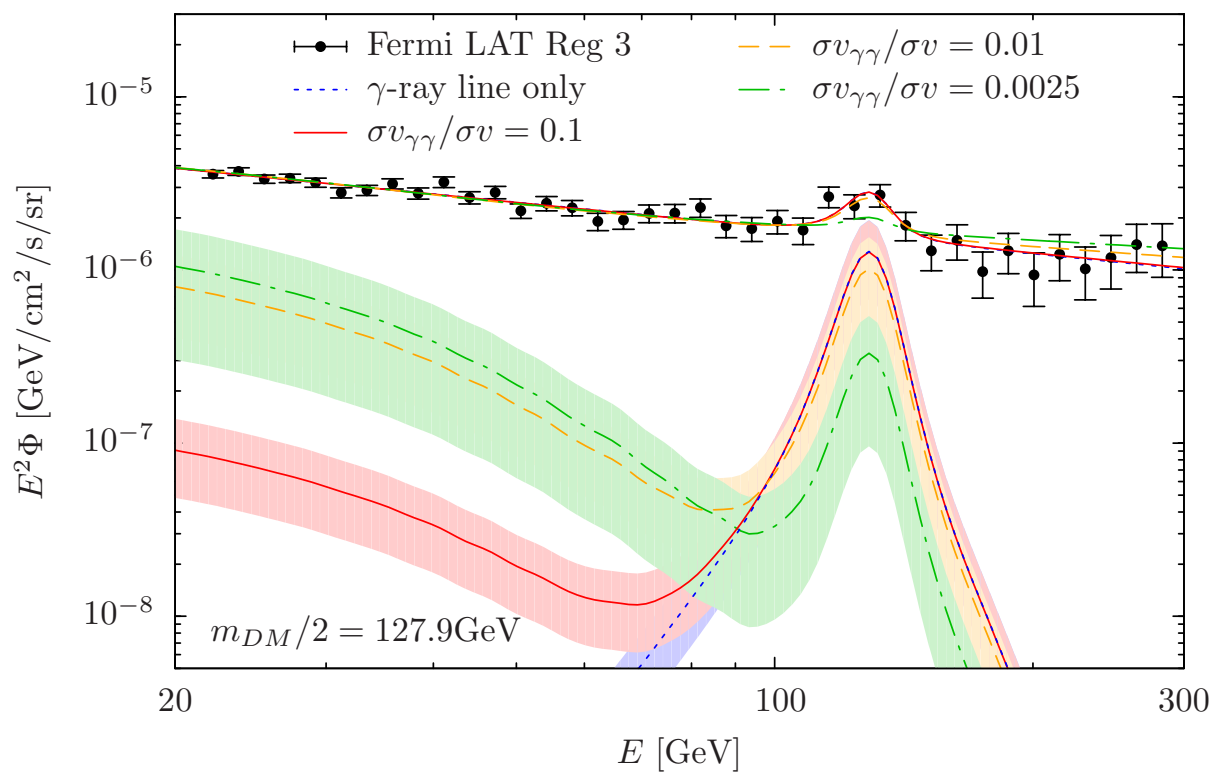

Annihilating Dark Matter (ULTRACLEAN)

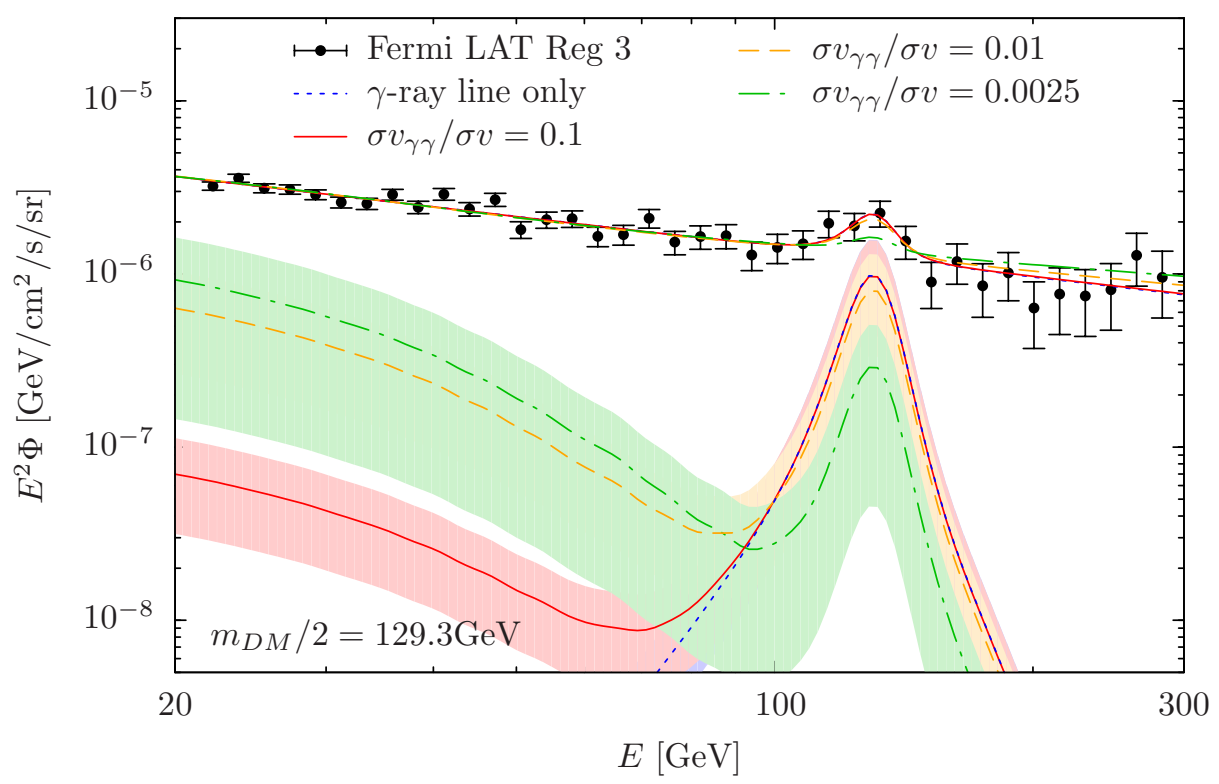

Figure 3: Sum of monochromatic and continuum photon flux originating from dark matter annihilating into $\gamma \gamma$ and $W^{+} W^{-}$with branching ratios $\mathrm{BR}_{\gamma}=0.1,0.01$, and 0.0025 , respectively. The lower lines show the contribution from dark matter annihilation, and the upper lines the sum of signal and fitted power-law background. The dashed blue line shows the flux when taking the line only into account. The Fermi data are the same as in Fig. 1. 

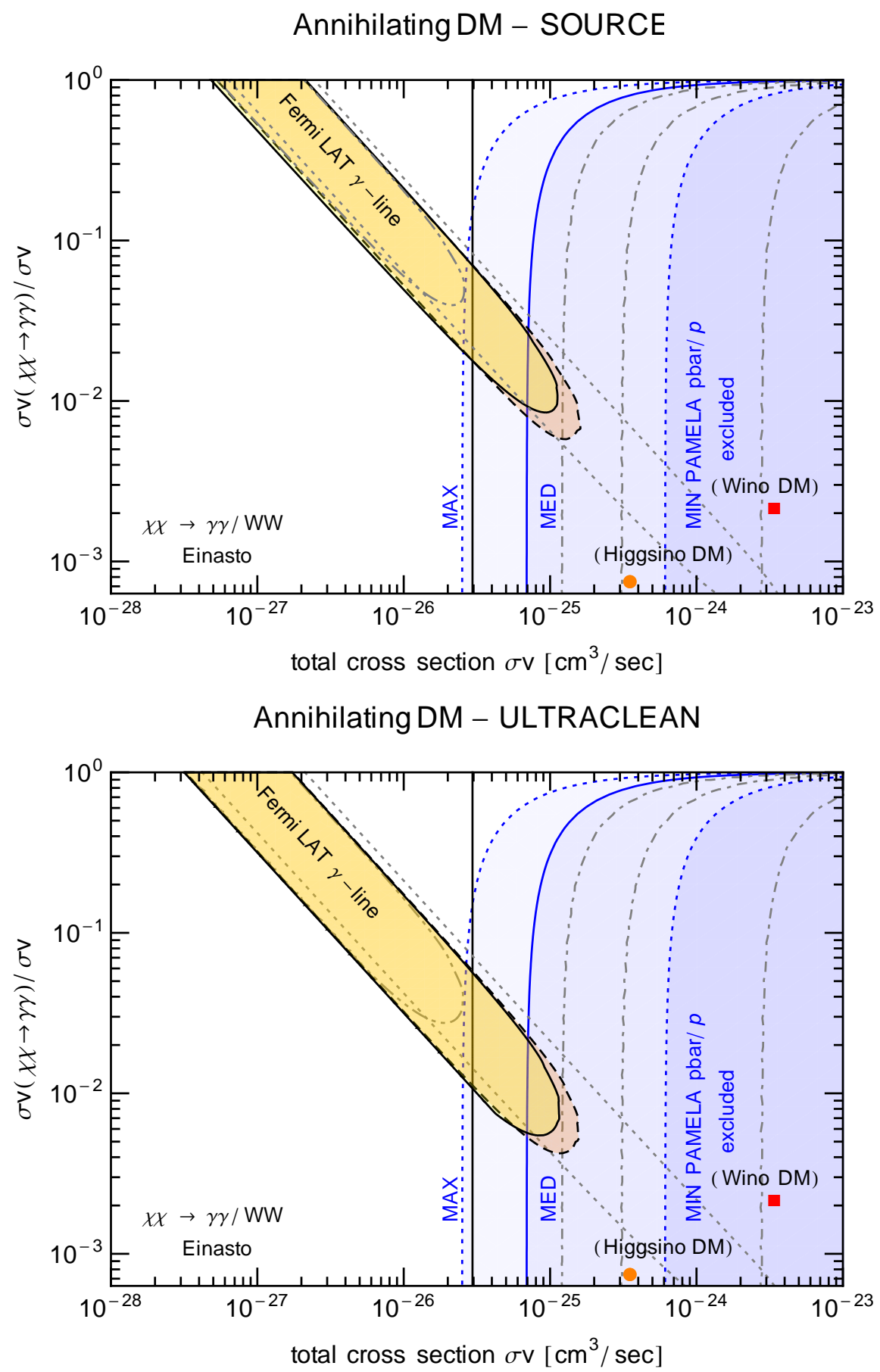

Figure 4: Combined fit of the monochromatic line at $\approx 130 \mathrm{GeV}$ as well as the continuum gamma-ray spectrum from dark matter annihilation into $\gamma \gamma$ and $W^{+} W^{-}$final states. The shaded areas correspond to 95\%C.L. regions (yellow/orange: Fermi-LAT Reg 3/4 best-fit region; blue: PAMELA $\bar{p} / p$ excluded region) for the total annihilation cross section $\sigma v$ and the branching ratio $\sigma v_{\gamma \gamma} / \sigma v$. The prediction from higgsino and wino dark matter (red and orange dots) is shown for illustration, as well as the thermal cross-section (straight black line). 


\begin{tabular}{ccc|ccc|cc}
\hline & $\mu$ & $M_{2}$ & $m_{\chi_{0}^{1}}$ & $m_{\chi_{0}^{2}}$ & $m_{\chi_{ \pm}^{1}}$ & $\sigma v_{\gamma \gamma}\left(\sigma v_{\gamma Z}\right)$ & $\sigma v_{W W}\left(\sigma v_{Z Z}\right)$ \\
\hline $\mathrm{H}$ & 139 & 1000 & 135.89 & 144.44 & 139.20 & $1.0(3.4) \cdot 10^{-28}$ & $2.1(1.4) \cdot 10^{-25}$ \\
$\mathrm{~W}$ & 400 & 143 & 139.79 & 408.08 & 139.94 & $2.0(10.9) \cdot 10^{-27}$ & $3.4(0.0) \cdot 10^{-24}$ \\
\hline
\end{tabular}

Table 3: Mass spectrum, annihilation cross sections and defining parameters for two scenarios with higgsino $(\mathrm{H})$ and wino-like (W) LSP. Particle masses are given in GeV, and cross sections in $\mathrm{cm}^{3} / \mathrm{s}$. The cross sections are rather insensitive to the other parameters, which we fixed to $A=0, M_{1,3}=\tilde{m}=1 \mathrm{TeV}$ and $\tan \beta=7$ for illustration. For the wino case the $\mu$ parameter was chosen such that the neutralino-chargino mass splitting is of the same order than the minimal splitting expected from loop corrections [34 in order to yield an estimate for the maximal possible gamma cross sections.

to $\sigma v_{\gamma Z} /\left(2 \sigma v_{\gamma \gamma}\right)=1.68$ as well as $\sigma v_{Z Z} / \sigma v_{W W}=0.66$. However, we freely vary the weighted branching ratio $\mathrm{BR}_{\gamma}=\left(\sigma v_{\gamma \gamma}+0.5 \sigma v_{\gamma Z}\right) / \sigma v_{t o t}$. In this case we obtain the best fit for dark matter masses in the range $132-137 \mathrm{GeV}$, depending on the search region and the data sample, and with similar significance than for one line. The resulting 95\% C.L. regions are shown in Fig. 5, which are very similar to the case of a single line discussed before, indicating that the lower limit $\mathrm{BR}_{\gamma} \gtrsim 0.4-0.8 \%$ is rather insensitive to the detailed assumptions about the cross sections of the various annihilation channels.

Finally, we briefly remark that both higgsino and wino-like dark matter can be ruled out as an explanation for the tentative gamma line based on the large continuum photon flux obtained in both cases. The cross sections for two representative scenarios are given in Tab. 3, leading to $\mathrm{BR}_{\gamma} \sim 0.08 \%$ (0.2\%) for higgsino(wino)-like dark matter. Even when allowing for a boost factor or a mixed dark matter scenario in order to achieve a line flux of the required size, the continuum flux overshoots the measured flux at energies around $20 \mathrm{GeV}$, which is illustrated in Fig. 6. In particular, we remark that this conclusion is independent of the dark matter distribution, and therefore of the uncertainties associated with it. According to the recent analysis in [35], electroweak corrections could even further reduce the $\gamma \gamma$ and $\gamma Z$ cross sections for low-mass wino dark matter by a sizeable amount. 

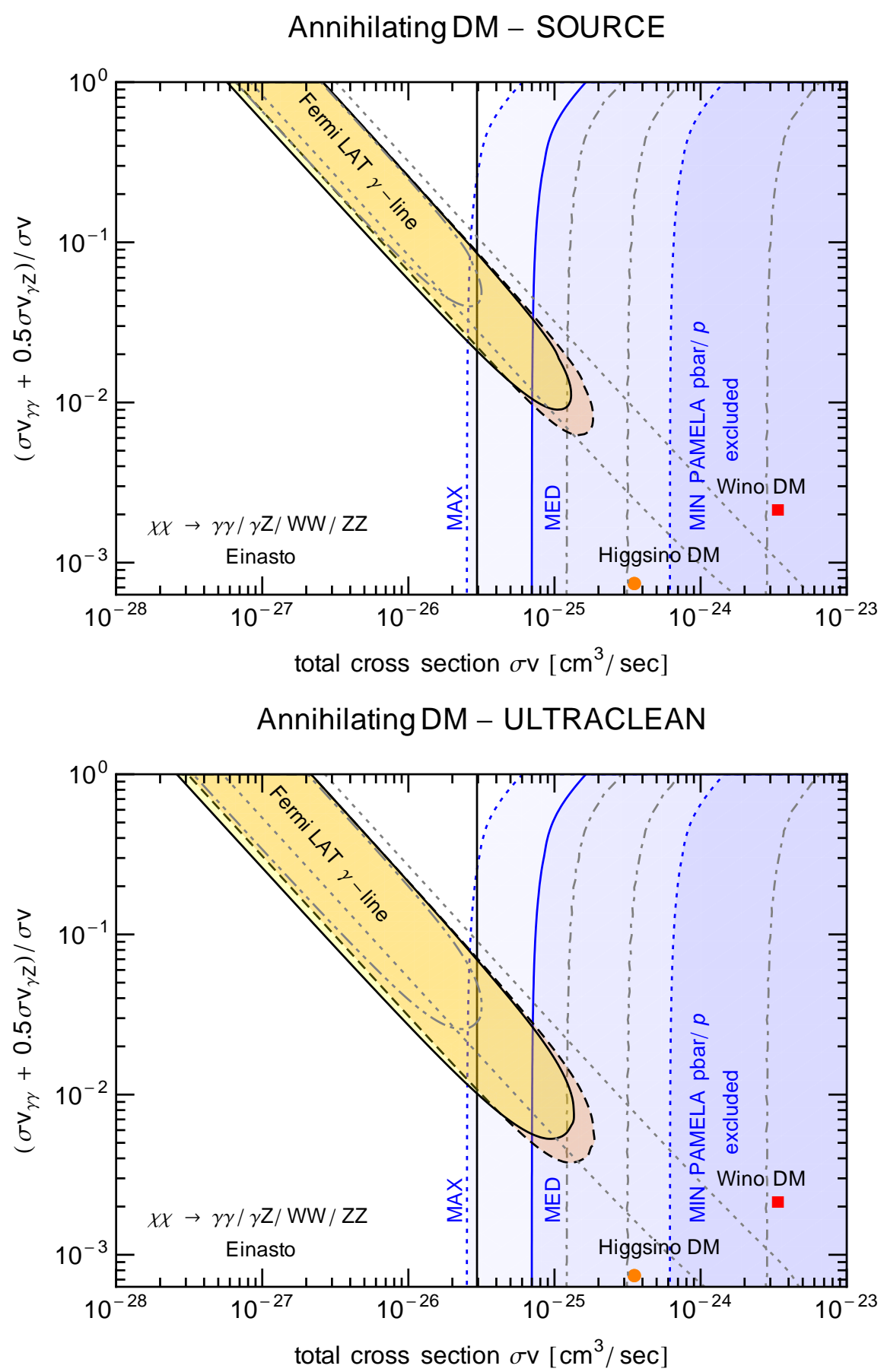

Figure 5: As Fig.4 4 , but for a model with two lines from annihilation into $\gamma \gamma$ and $\gamma Z$, as well as continuum photons from annihilations into $W^{+} W^{-}$and $Z Z$ final states. The axes correspond to the total cross section $\sigma v$ and to the weighted ratio $\left(\sigma v_{\gamma \gamma}+0.5 \sigma v_{\gamma Z}\right) / \sigma v$, respectively. For the fit we fixed the ratios $\sigma v_{\gamma Z} /\left(2 \sigma v_{\gamma \gamma}\right)=1.68$ and $\sigma v_{Z Z} / \sigma v_{W W}=0.66$ motivated by the higgsino dark matter scenario. The actual prediction from higgsino and wino dark matter is also shown (red and orange dots). 
Higgsino Dark Matter

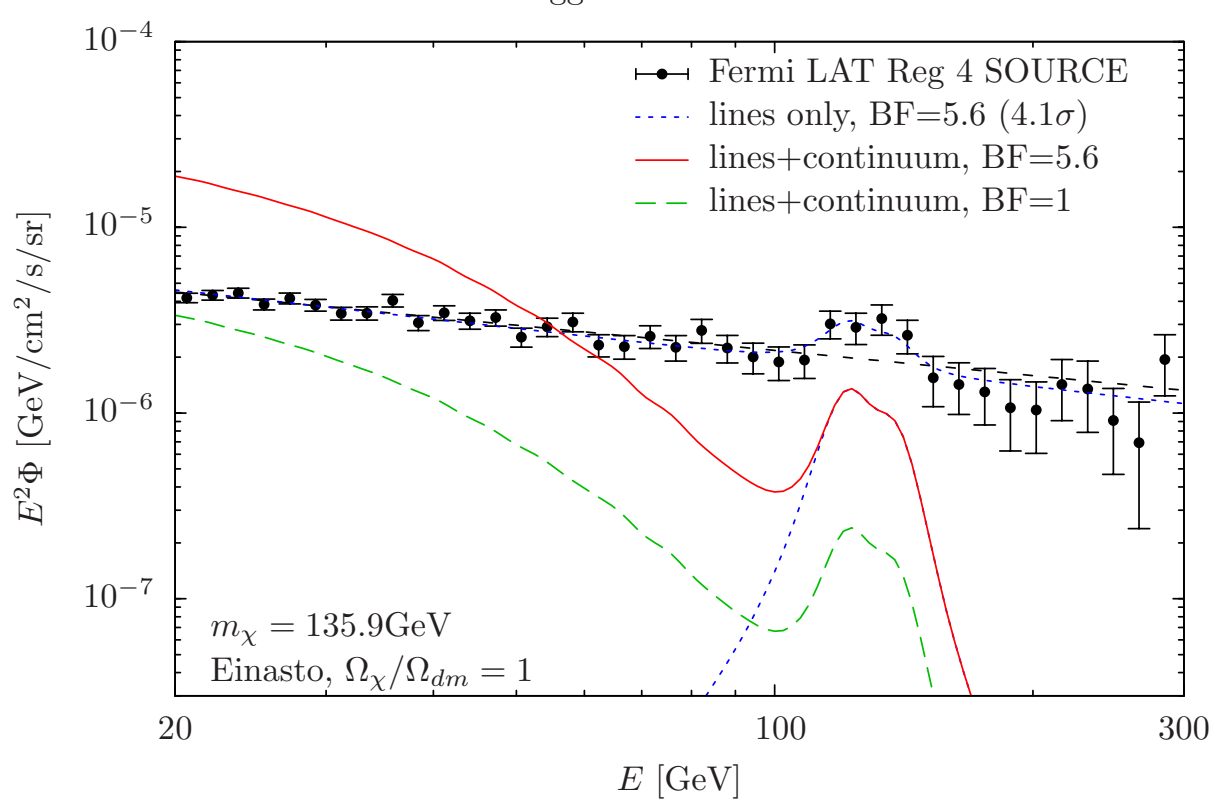

Wino Dark Matter

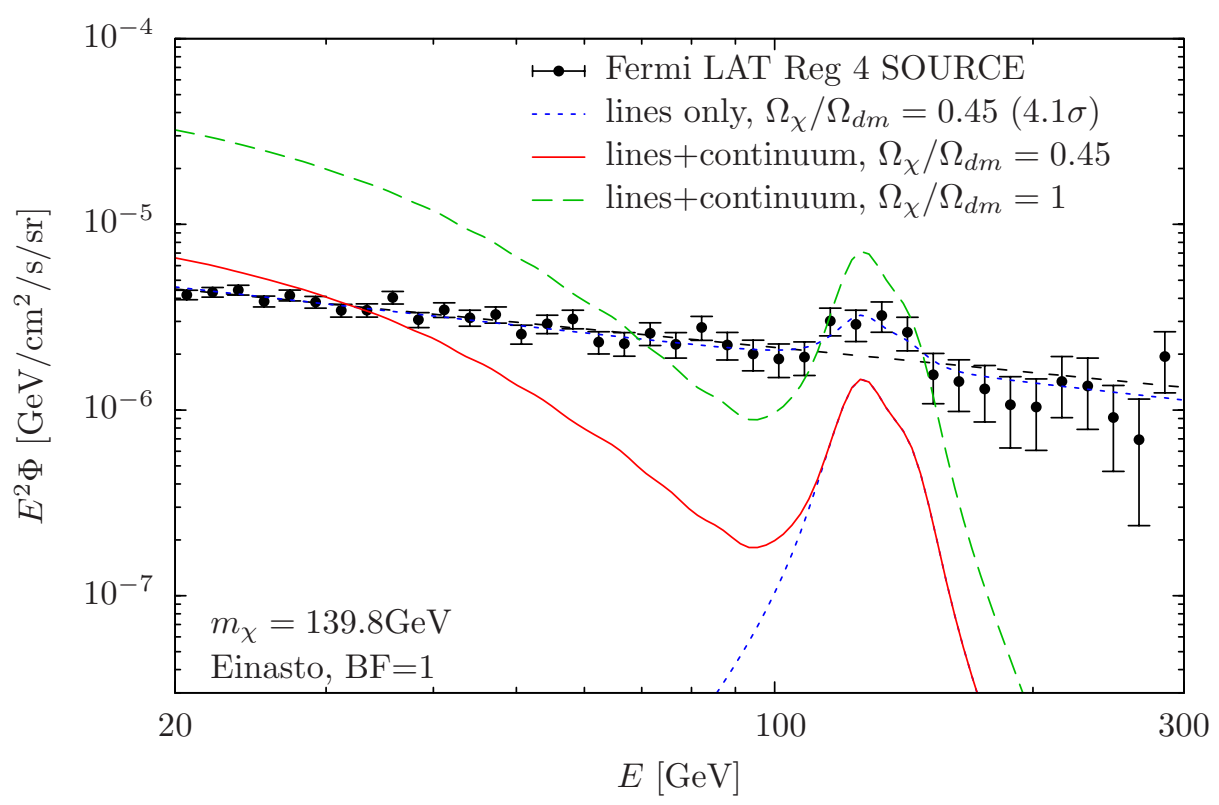

Figure 6: Predicted gamma-ray flux for higgsino dark matter (upper graph) and wino dark matter (lower graph, green dashed lines). The monochromatic gamma-ray flux is too small(large) in the higgsino(wino) case for explaining the Fermi excess at $\approx 130 \mathrm{GeV}$ when assuming an Einasto profile. We also show the flux obtained for a boost factor $B F=5.6$ in the higgsino case and when assuming that the wino accounts only for $45 \%$ of the dark matter density, respectively (red solid lines). In both cases, the monochromatic contribution to the flux would yield a good fit to the Fermi data (blue dotted lines). However, the continuum contribution to the gamma-ray flux overshoots the measured flux at lower energies, and therefore both scenarios can be ruled out. The Fermi data are taken from [12]. 


\section{Constraints from antiprotons}

Constraints that are complementary to the continuum gamma-ray spectrum arise from the production of antiprotons in the decay and fragmentation of the weak gauge bosons, Higgs bosons or quarks produced in dark matter annihilation or decay. The resulting primary flux of antiprotons can be potentially observed in the cosmic ray spectrum measured at the Earth. Antiprotons are produced with a rate per unit of kinetic energy and unit volume at a position $r$ with respect to the center of the Galaxy given by

$$
Q_{\bar{p}}(E, r)=\left\{\begin{array}{c}
\frac{\rho_{d m}(r)}{m_{D M}} \frac{1}{\tau} \\
\frac{\rho_{d m}(r)^{2}}{2 m_{D M}^{2}} \sigma v_{t o t}
\end{array}\right\} \times \sum_{f} \mathrm{BR}_{f} \frac{d N_{f}^{\bar{p}}}{d E} \quad \text { for } \quad\left\{\begin{array}{l}
\text { decay } \\
\text { annihilation }
\end{array}\right.
$$

where $d N_{f}^{\bar{p}} / d E$ is the number of antiprotons per kinetic energy and per decay produced in the annihilation/decay channels $f$ with branching ratio given by $\mathrm{BR}_{f}$, and $\rho_{d m}(r)$ is the dark matter density profile.

The antiproton-to-proton flux ratio observed by the PAMELA satellite experiment [36] agrees well with the expectation for the secondary antiproton spectrum produced by spallation of ordinary cosmic rays on the interstellar medium. Therefore, upper limits on a possible primary component from dark matter annihilation or decay can be derived (see e.g. $37-42]$ ). The main source of uncertainty enters due to the propagation of antiprotons in the Galactic magnetic field from the production point to the Earth. In order to obtain lower limits on the dark matter lifetime and upper limits on the annihilation cross section we follow here the approach described in detail in Refs. 40 , 42]. In particular, we describe the propagation by a cylindrical two-zone diffusion model using three representative sets of parameters leading to minimum, medium and maximum antiproton fluxes consistent with the charged cosmic ray abundances [43]. In order to obtain conservative limits we use the minimal secondary antiproton spectrum from Refs. [40, 44], and use a lower cut of $T>1.5 \mathrm{GeV}$ for the kinetic energy.

For the case of dark matter decaying into $Z \nu, h \nu, W \ell$ with the same branching ratios used above, the 95\%C.L. lower limits on the dark matter lifetime is also shown in Fig. 2 as blue shaded regions, for the three sets of propagation parameters and the Einasto dark matter profile. For a branching ratio much smaller than one, the 95\%C.L. lower limit on the lifetime is given by $\tau \gtrsim(0.29,1.34,3.31) \times 10^{27} \mathrm{~s}$ for the minimum, medium and maximum propagation models, respectively. Compared to the uncertainty arising 
from the propagation model, the dependence of the lower limit on the dark matter profile is rather weak. As can be seen in Fig. 2, the constraints from antiprotons require the branching ratio to lie above $\sim \mathcal{O}(1 \%, 5 \%, 10 \%)$, depending on the propagation model, if we require that the partial lifetime into monochromatic photons accounts for the gamma feature at $130 \mathrm{GeV}$. Even for the minimal propagation model, these lower limits on the branching ratio are more stringent than the ones obtained from the continuum gamma-ray flux. However, they are also more dependent on astrophysical uncertainties related to propagation as well as the secondary antiproton flux [41]. For comparison, we also show the lower limits on the lifetime obtained from just requiring that the primary antiproton flux does not overshoot the observed antiproton-to-proton ratio. The resulting limits are somewhat weaker, $\tau \gtrsim(0.64,2.81,6.62) \times 10^{26}$ s. For the most conservative case, i.e. for minimal propagation, the resulting lower limit on the branching ratio is comparable to the one obtained from the continuum gamma-ray flux (see Fig. 2).

For the case of annihilating dark matter into $W^{+} W^{-}$, the antiproton constraints shown in Fig. 4 correspond to upper limits on the annihilation cross section $\sigma v \lesssim$ $(6.1,0.69,0.25) \times 10^{-25} \mathrm{~cm}^{3} / \mathrm{s}$ when taking the secondary flux into account. The resulting lower limits on the branching ratio $\sigma v_{\gamma \gamma} / \sigma v$ are more stringent or comparable to the ones from the continuum flux for maximum or medium propagation, respectively, as can be seen in Fig 4. In the conservative case, when taking only the primary flux into account, one obtains rather weak limits $\sigma v \lesssim(2.8,0.31,0.12) \times 10^{-24} \mathrm{~cm}^{3} / \mathrm{s}$ that are comparable to the continuum flux only in the maximum case. The antiproton constraints are very similar for the case when dark matter annihilates into $\gamma \gamma / \gamma Z / W W / Z Z$ final states (see Fig. 5), except for the antiprotons from $\gamma Z$ that contribute for very large branching ratios.

Altogether, we find that antiproton constraints are typically the leading ones for decaying dark matter, while the continuum gamma-ray flux is more or equally important for annihilating dark matter.

\section{Morphology of the Fermi excess at $130 \mathrm{GeV}$}

Apart from constraints arising from the gamma-ray spectrum or from antiprotons, the Fermi-LAT data also provide valuable information about the spatial distribution of the gamma-ray flux observed towards the Galactic center (the angular resolution 
of the LAT is $\Delta \theta \sim 0.2^{\circ}[12]$ ). The hints for a feature in the spectrum are based on about 69(57) photons collected within 43 months inside search regions 3(4) (solid angle $\Delta \Omega / 4 \pi=0.025(0.015))$ defined in $[12]$ and the SOURCE sample, and 53(46) for the ULTRACLEAN sample. Consequently, any information about the detailed spatial distribution of the excess within these regions is at present necessarily limited by statistics. Nevertheless, the amount of data allows to test the compatibility with certain spatial distributions. In particular, in the analysis of [19] it was found that using a Gaussian template the excess appears to originate from a rather small region $\approx 3^{\circ} \mathrm{FWHM}$, possibly with a small offset from the Galactic center of $1.5^{\circ}$ along the Galactic plane. Additionally, it was argued that an Einasto or contracted NFW profile yields an even better description, when also taking an offset into account.

In the following, we leave aside the question about the origin of a possible offset in connection with a dark matter explanation. Instead, under the tentative hypothesis that dark matter is indeed responsible for the Fermi feature, we discuss the impact of the apparent morphology on decaying versus annihilating dark matter. The flux of photons arriving at the Earth per unit of energy and solid angle from dark matter decay or annihilation is given by the line-of-sight integral over the dark matter density or the density squared, respectively, along the direction of observation (see e.g. [46]),

$$
\frac{d J_{\gamma}}{d E d \Omega}=\frac{1}{4 \pi} \delta\left(E-E_{\gamma}\right)\left\{\begin{array}{l}
\frac{1}{\tau_{\gamma \nu} m_{D M}} \int \frac{\rho}{\text { l.o.s. }} d s \rho_{d m}(r) \text { decay } \\
\frac{2 \sigma v_{\gamma \gamma}}{m_{D M}^{2}} \int \frac{1}{\text { l.o.s. }} d s \frac{1}{2} \rho_{d m}(r)^{2} \text { annihilation }
\end{array}\right.
$$

where $r=\sqrt{\left(r_{0}-s \cos \xi\right)^{2}+(s \sin \xi)^{2}}, r_{0} \simeq 8.5 \mathrm{kpc}$ is the distance of the Earth from the Galactic center, and $\xi$ the angle measured with respect to the direction towards the center of the Galaxy. As a reference, we consider the density profiles given, respectively, by a generalized Navarro-Frenk-White (NFW) [47] or Einasto profile [48],

$$
\rho_{d m}(r) \propto \frac{1}{\left(r / r_{s}\right)^{\alpha}\left(1+r / r_{s}\right)^{3-\alpha}}, \exp \left(-\frac{2}{\alpha_{E}}\left(r / r_{s}\right)^{\alpha_{E}}\right),
$$

with $\alpha_{E}=0.17, \alpha=1$ and scale radius $r_{s}=20 \mathrm{kpc}$. We also consider two strongly peaked distributions with $\alpha=(1.3,1.7)$, that may arise from adiabatic contraction. All profiles are normalized such that the local density is $\rho_{0}=0.4 \mathrm{GeV} / \mathrm{cm}^{3}$, except for $\alpha=1.7$ where we use $\rho_{0}=0.28 \mathrm{GeV} / \mathrm{cm}^{3}$ corresponding to the maximal allowed $1 \sigma$-value when taking observational constraints from microlensing and measurements of the Galactic dynamics into account [49]. 
Spatial distribution $(124.7-133.4 \mathrm{GeV})$

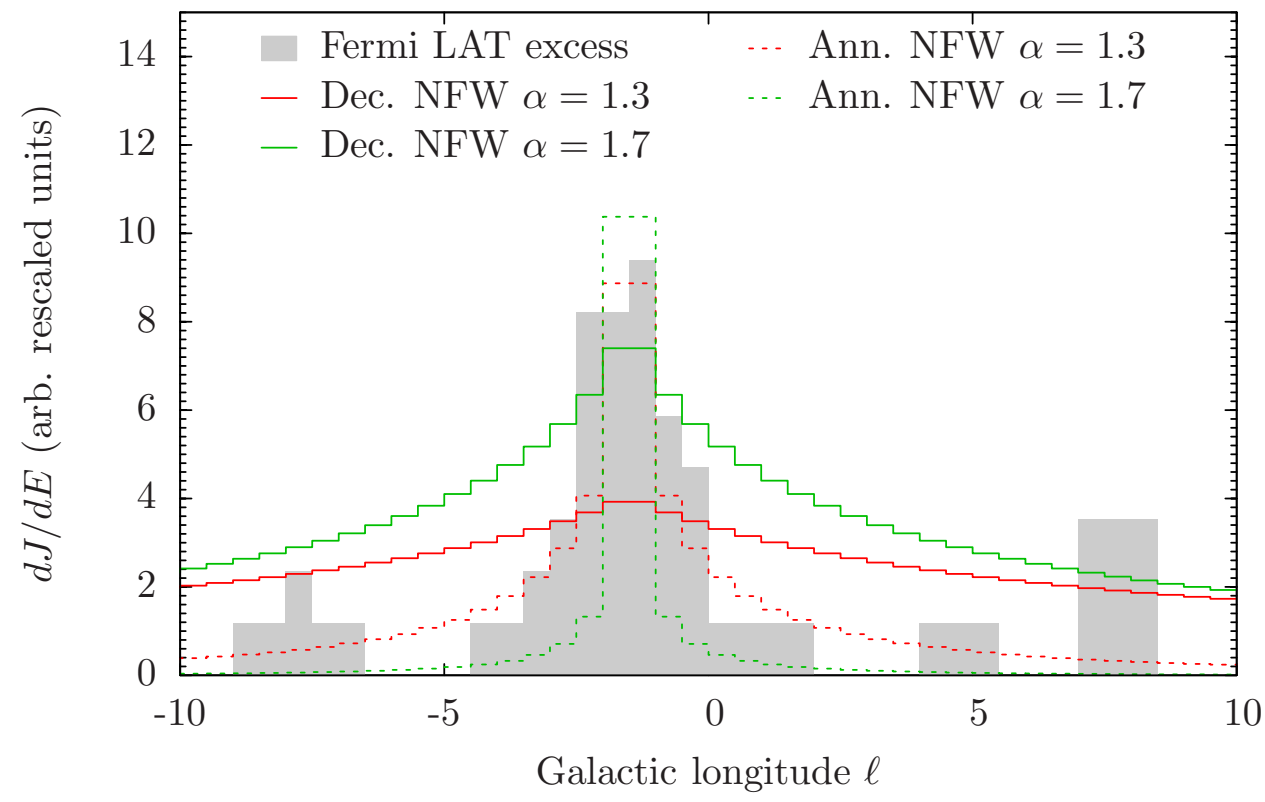

Spatial distribution $(124.7-133.4 \mathrm{GeV})$

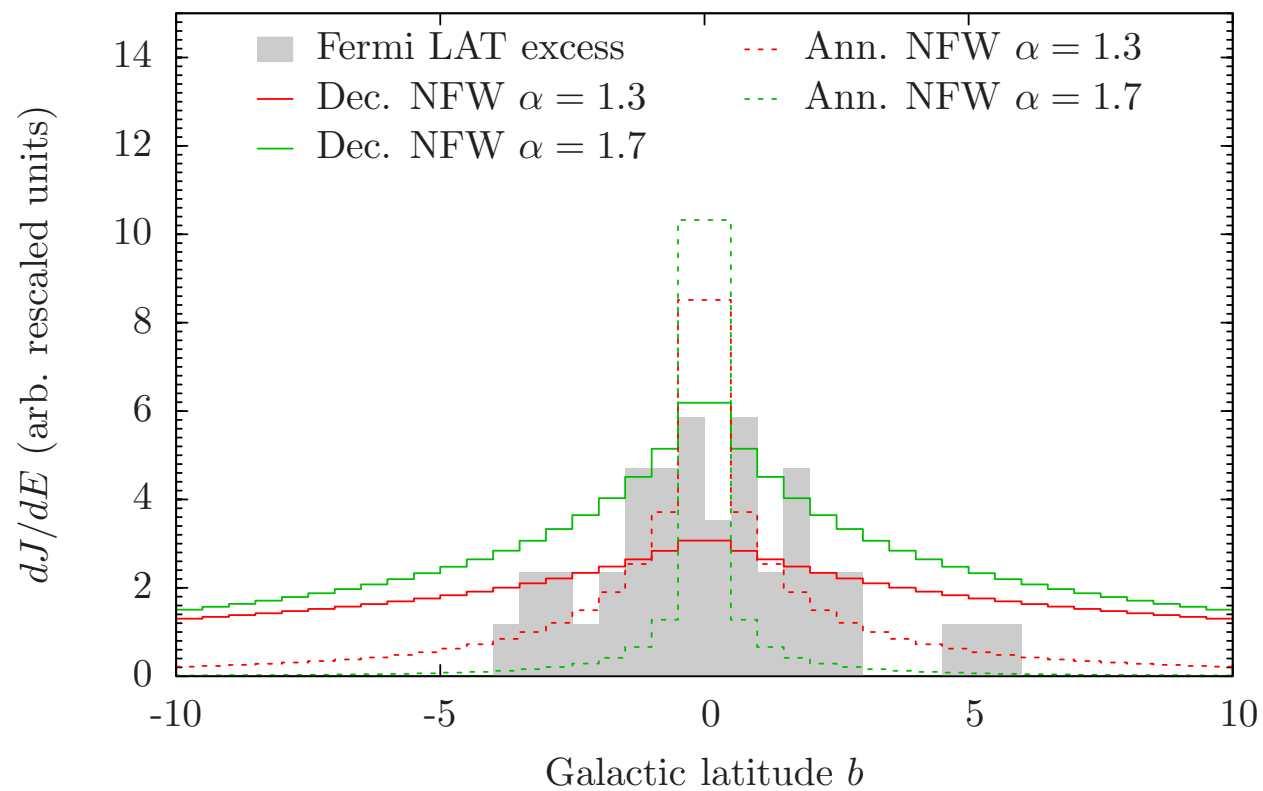

Figure 7: Expected spatial profile of the gamma-ray emission from sections along the Galactic plane $\left(\Delta \ell=0.5^{\circ},|b|<5^{\circ}\right.$, upper graph) and perpendicular to the plane $\left(\Delta b=0.5^{\circ},-5^{\circ}<\right.$ $\ell<-2^{\circ}$, lower graph) for decaying and annihilating dark matter and two density profiles centered around $(\ell, b)=\left(-1.5^{\circ}, 0\right)$. For illustration, we also show the count maps indicating the shape of the Fermi-LAT excess in the energy range 124.7-133.4 GeV taken from Ref. [19]. 
In Ref. [19] it has been argued that the morphology of the Fermi excess at $\approx 130 \mathrm{GeV}$ is consistent with dark matter annihilation assuming the Einasto or NFW profile, possibly with $\alpha$ slightly larger than one. In order to obtain a similar emission profile for decaying dark matter, a much more peaked dark matter distribution would be necessary. In order to illustrate this point, we show in Fig. 7 the expected spatial distribution for the contracted NFW profiles. For comparison we also show the corresponding shape for annihilating dark matter, and the count maps taken from [19]. Qualitatively, it seems that even for the strongly contracted profile with $\alpha=1.7$ the expected distribution for decaying dark matter is too flat, especially for the profile along the Galactic plane. One may speculate that, in the perpendicular direction, the excess could be less peaked. However, a definite statement is presently impossible due to statistical limitations.

For decaying dark matter, one would expect also a comparably large signal arising from the Galactic halo, where no excess has been observed. Therefore, it is instructive to compare the halo limit with the required lifetime to explain the excess in the central region. The partial dark matter lifetimes $\tau_{\gamma \nu}$ that are required in order to explain the feature in the Fermi gamma-ray spectrum present within various search regions defined in [12], and for the various assumptions of the radial dark matter distribution, are shown in Fig. 8. Although the best-fit values for the considered search regions are compatible with each other, they are in tension with the lower limits on the lifetime obtained by the recent analysis of the Fermi collaboration [9], also shown in Fig. 8, Note that the Fermi analysis is based on a rather wide search region, and therefore sensitive to the dark matter flux originating in the halo of the Milky Way. Due to the linear scaling of the flux with the dark matter density in the case of decaying dark matter, one typically expects a larger flux from the halo compared to the center than for annihilating dark matter. Thus, for the case of decaying dark matter the gamma-ray line should have been observed also within the search region used by the Fermi collaboration, provided that the radial distribution of dark matter can be well described by the considered NFW or Einasto profiles. The discrepancy is less pronounced for the contracted profiles which are more cuspy towards the Galactic center. On the other hand, for annihilating dark matter, the cross section required to explain the Fermi gamma-ray feature is compatible with earlier upper limits assuming NFW or Einasto profiles [12].

Clearly, this tension disfavors the explanation of the excess in terms of decaying dark matter. However, because of the large uncertainty of the actual dark matter distribution close to the Galactic center as compared to the halo [2, 16, 50], and since 

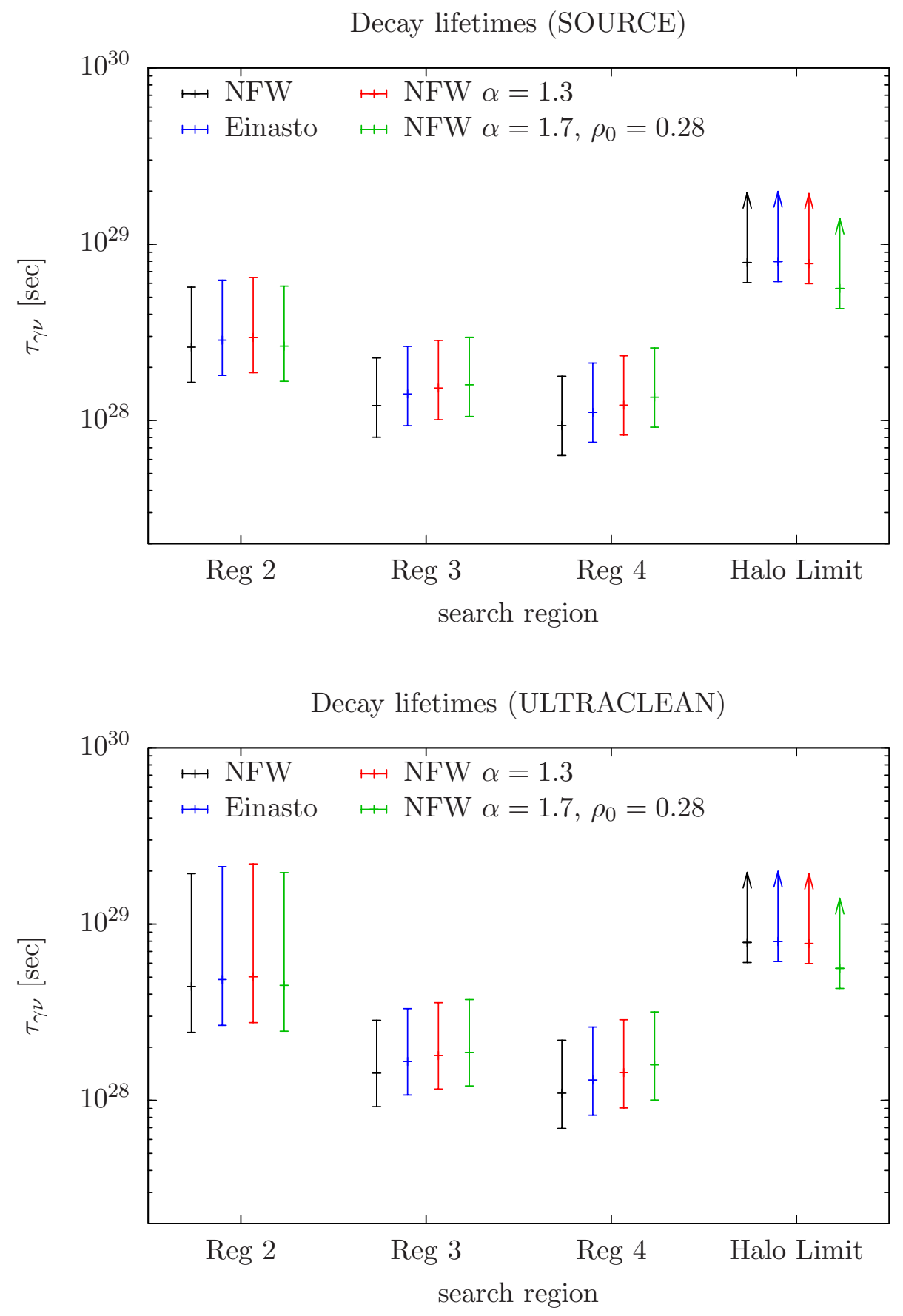

Figure 8: Best fit values for the partial dark matter lifetime $\tau_{\gamma \nu}$ for a decay into monochromatic photons, obtained for the search regions 2, 3 and 4 defined in Ref. [12], for various dark matter profiles. The error bars show the 95\%C.L. regions. Also shown are the lower limits on the partial lifetime from the most recent Fermi LAT analysis (the lower error bars include the estimated systematic uncertainty) [9], that are based on a wide search region (Halo Limit). The upper and lower figures correspond to the two Fermi data samples used in 12 . 
one cannot exclude large systematic uncertainties at present [9], we believe that it would be premature to rule out decaying dark matter as an explanation of the Fermi excess based on this tension.

\section{Conclusion}

In light of tentative evidence for a gamma-ray line observed by Fermi-LAT near the Galactic center, we have investigated the consequences for several prototype scenarios of decaying and annihilating dark matter, motivated by supersymmetric models with gravitino, higgsino or wino-like LSP. We find that, independently of the actual dark matter distribution, the consistency of continuum and monochromatic contributions to the photon spectrum from dark matter decay or annihilation requires a branching ratio into monochromatic photons larger than $\mathrm{BR}_{\gamma} \gtrsim 0.5 \%$. Both higgsino and wino dark matter can be ruled out because of a too large continuum flux, independently of the production mechanism, while gravitino dark matter with wino NLSP is compatible. We have also investigated constraints arising from the primary antiproton flux, which depend on the adopted propagation model. The resulting limits are comparable to the ones from continuum gamma-rays for annihilating dark matter, but can be more important for decaying dark matter. The morphology of the tentative excess, if confirmed, could yield valuable information on the distribution of dark matter close to the Galactic center. When assuming conventional NFW or Einasto profile functions, the required partial lifetimes for decaying dark matter $\tau_{\gamma \nu} \sim(1-3) \times 10^{28} \mathrm{~s}$ are in tension with lower limits obtained from the Galactic halo. This tension is less pronounced for more cuspy contracted profiles. If decaying gravitino dark matter is indeed responsible for the excess, the dark matter density should be enhanced in the Galactic center region compared to conventional models.

\section{Acknowledgements}

The authors thank S. Bobrovskyi, T. Bringmann, L. Covi, J. Hajer, A. Ibarra, A. Morselli, A. Ringwald and C. Weniger for helpful discussions. This work has been supported by the German Science Foundation (DFG) within the Collaborative Research Center 676 "Particles, Strings and the Early Universe". 


\section{Appendix}

In the following we collect the formulae needed to evaluate the various branching ratios of gravitino LSP decays in supersymmetric models with bilinear R-parity breaking.

The couplings of the gravitino field $\psi_{\nu}$ to matter and gauge fields fields are given by 51,52 ,

$$
\mathcal{L}=\frac{i}{\sqrt{2} M}\left(\bar{\chi} \gamma^{\nu} \gamma^{\mu}\left(D_{\mu} \phi\right) \psi_{\nu}+\text { c.c. }\right)-\frac{1}{4 M} \bar{\lambda} \gamma^{\nu} \sigma^{\mu \rho} \psi_{\nu} F_{\mu \rho} .
$$

Here $\chi$ stands for the left-handed lepton and higgsino doublets $l, h_{u}, h_{d}$, and $\phi$ for the corresponding scalar leptons and Higgs fields $\tilde{l}, H_{u}, H_{d} ; F_{\nu \rho}$ denotes the fields strengths of $U(1)_{Y}$ and $S U(2)$ gauge interactions, $B_{\nu \rho}$ and $W_{\nu \rho}^{I}$, and $\lambda$ the corresponding gauginos $b$ and $w^{I}$.

In the case of bilinear R-parity breaking the transition from weak to mass eigenstates has been worked out in detail in [53,54]. Inserting these field transformations in Eq. (5.1) and shifting the Higgs fields around their vacuum expectation values, one finds for the R-parity breaking gravitino couplings

$$
\begin{aligned}
\mathcal{L}_{3} \supset \frac{i}{\sqrt{2} M_{\mathrm{P}}}\{ & \left(\kappa_{h i} \partial_{\mu} h+i \kappa_{z i} m_{Z} Z_{\mu}\right) \bar{\nu}_{i} \gamma^{\nu} \gamma^{\mu} \psi_{\nu}+i \sqrt{2} \kappa_{w i} m_{W} W_{\mu}^{-} \bar{e}_{i} \gamma^{\nu} \gamma^{\mu} \psi_{\nu} \\
& +i m_{Z}\left(\xi_{z i} \partial_{\mu} Z_{\nu}+\xi_{\gamma i} \partial_{\mu} A_{\nu}\right) \bar{\nu}_{i} \gamma^{\lambda} \sigma^{\mu \nu} \psi_{\lambda} \\
& \left.+i \sqrt{2} m_{W} \xi_{w i} \partial_{\mu} W_{\nu}^{-} \bar{e}_{i} \gamma^{\lambda} \sigma^{\mu \nu} \psi_{\lambda}\right\}+ \text { h.c. }
\end{aligned}
$$

The couplings $\kappa$ and $\xi$ are determined by the R-parity breaking parameters $\zeta_{i}$, the gaugino masses $M_{1,2}$ and the $\mu$ parameter [53,54],

$$
\begin{aligned}
\kappa_{h i} & =\frac{\zeta_{i}}{\sqrt{2}}\left(1-\sin 2 \beta \frac{m_{Z}^{2}\left(M_{1} \cos ^{2} \theta_{W}+M_{2} \sin ^{2} \theta_{W}\right)}{M_{1} M_{2} \mu}\right) \\
\kappa_{z i} & =\frac{\zeta_{i}}{\sqrt{2}}\left(1+\sin 2 \beta \frac{m_{Z}^{2}\left(M_{1} \cos ^{2} \theta_{W}+M_{2} \sin ^{2} \theta_{W}\right)}{M_{1} M_{2} \mu}\right) \\
\kappa_{w i} & =\frac{\zeta_{i}}{\sqrt{2}}\left(1-\sin 2 \beta \frac{m_{W}^{2}}{M_{2} \mu}\right) \\
\xi_{\gamma i} & =\frac{\zeta_{i}}{\sqrt{2}} \cos \theta_{W} \sin \theta_{W} \frac{m_{Z}\left(M_{2}-M_{1}\right)}{M_{1} M_{2}} \\
\xi_{z i} & =-\frac{\zeta_{i}}{\sqrt{2}} \frac{m_{Z}\left(M_{2} \sin ^{2} \theta_{W}+M_{1} \cos ^{2} \theta_{W}\right)}{M_{1} M_{2}} \\
\xi_{w i} & =-\frac{\zeta_{i}}{\sqrt{2}} \frac{m_{Z}}{M_{2}},
\end{aligned}
$$


where $\theta_{W}$ is the weak angle. For a gravitino LSP one has $M_{1}, M_{2}, \mu \gtrsim m_{3 / 2}$. Note that the gauge boson couplings $\xi$ satisfy the relation

$$
\xi_{Z i}+\tan \theta_{\mathrm{W}} \xi_{\gamma i}=\xi_{W i}
$$

The gravitino couplings (5.2) have previously been obtained in an effective Lagrangian approach 392 , The couplings $\xi=\mathcal{O}\left(m_{Z} / m_{3 / 2}\right)$ correspond to dimension-6 operators and the leading terms of the couplings $\kappa$ to a dimension- 5 operator, which gives

$$
\Gamma\left(\psi_{3 / 2} \rightarrow h \nu_{i}\right) \simeq \Gamma\left(\psi_{3 / 2} \rightarrow Z \nu_{i}\right) \simeq \frac{1}{2} \Gamma\left(\psi_{3 / 2} \rightarrow W^{+} e_{i}^{-}\right)
$$

for $m_{Z} / m_{3 / 2} \ll 1$. The subleading contributions $\mathcal{O}\left(m_{Z}^{2} / m_{3 / 2}^{2}\right)$ to $\kappa$ correspond to dimension-7 operators which were not considered in [39].

Based on the calculations of [55,56 one can now obtain the partial two-body gravitino decay widths by matching coefficients. The result reads

$$
\begin{aligned}
\Gamma\left(\psi_{3 / 2} \rightarrow h \nu_{i}\right) & =\frac{m_{3 / 2}^{3}}{384 \pi M_{\mathrm{P}}^{2}} \kappa_{h i}^{2} \beta_{h}^{4} \\
\Gamma\left(\psi_{3 / 2} \rightarrow \gamma \nu_{i}\right) & =\frac{m_{3 / 2}^{3}}{64 \pi M_{\mathrm{P}}^{2}}\left|\xi_{\gamma i}\right|^{2} \\
\Gamma\left(\psi_{3 / 2} \rightarrow Z \nu_{i}\right) & =\frac{m_{3 / 2}^{3}}{384 \pi M_{\mathrm{P}}^{2}} \beta_{Z}^{2}\left(\kappa_{z i}^{2} H_{Z}+16 \frac{m_{Z}}{m_{3 / 2}} \operatorname{Re}\left(\kappa_{z i} \xi_{z i}\right) G_{Z}+6\left|\xi_{z i}\right|^{2} F_{Z}\right) \\
\Gamma\left(\psi_{3 / 2} \rightarrow W^{+} e_{i}^{-}\right) & =\frac{m_{3 / 2}^{3}}{192 \pi M_{\mathrm{P}}^{2}} \beta_{Z}^{2}\left(\kappa_{w i}^{2} H_{W}+16 \frac{m_{W}}{m_{3 / 2}} \operatorname{Re}\left(\kappa_{w i} \xi_{w i}\right) G_{W}+6\left|\xi_{W i}\right|^{2} F_{W}\right)
\end{aligned}
$$

The functions $\beta_{a}, H_{a}, G_{a}$ and $F_{a}(a=h, Z, W)$ are given by 55

$$
\begin{aligned}
\beta_{a} & =1-\frac{M_{a}^{2}}{m_{3 / 2}^{2}} \\
H_{a} & =1+10 \frac{M_{a}^{2}}{m_{3 / 2}^{2}}+\frac{M_{a}^{4}}{m_{3 / 2}^{4}}, \\
G_{a} & =1+\frac{1}{2} \frac{M_{a}^{2}}{m_{3 / 2}^{2}}, \\
F_{a} & =1+\frac{2}{3} \frac{M_{a}^{2}}{m_{3 / 2}^{2}}+\frac{1}{3} \frac{M_{a}^{4}}{m_{3 / 2}^{4}}
\end{aligned}
$$

\footnotetext{
${ }^{2}$ Note the change in notation compared to 39 .
} 


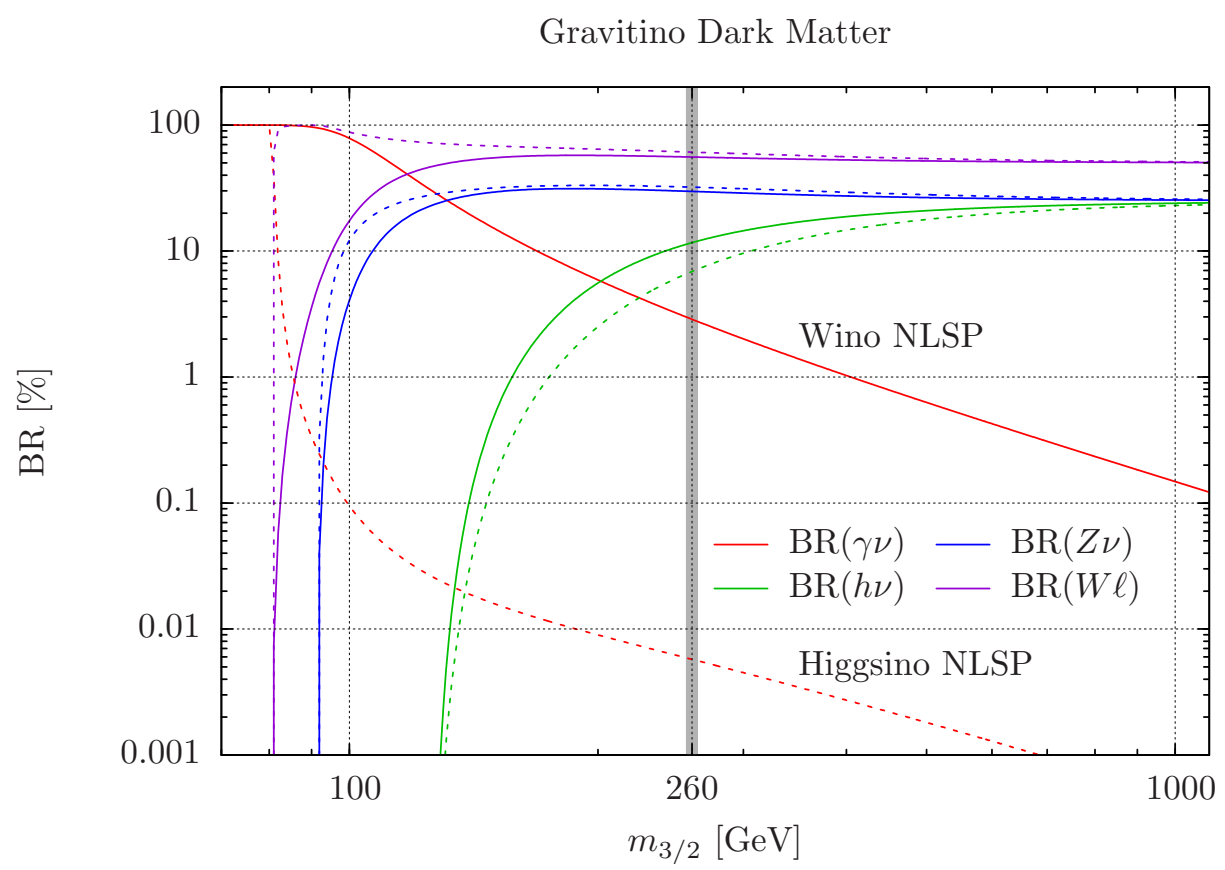

Figure 9: Branching ratios of two-body gravitino decays for two representative examples. Wino NLSP: $M_{2}=1.1 m_{3 / 2}, M_{1}=\mu=10 m_{3 / 2}$, and higgsino NLSP: $\mu=1.1 m_{3 / 2}$, $M_{1}=10 m_{3 / 2}, M_{2}=1.9 M_{1}$.

The decay width $\Gamma\left(\psi_{3 / 2} \rightarrow \gamma \nu\right)$ is of particular interest since it determines the strength of the gamma line at the end of the continuous spectrum. Neglecting corrections $\mathcal{O}\left(m_{Z}^{2} / m_{3 / 2}^{2}\right)$, one finds for the branching ratio

$$
\mathrm{BR}_{\gamma} \simeq \frac{3 \pi \alpha}{2 \sqrt{2} G_{F}} \frac{\left(M_{1}-M_{2}\right)^{2}}{M_{1}^{2} M_{2}^{2}}
$$

where $\alpha$ is the electromagnetic fine-structure constant and $G_{F}$ is Fermi's constant.

In Figure 9 the different branching ratios are shown as functions of the gravitino mass for the two representative cases of wino and higgsino NLSPs.

\section{References}

[1] L. Bergstrom and H. Snellman, Phys. Rev. D 37 (1988) 3737.

[2] For a review and references, see for example,

G. Bertone, D. Hooper and J. Silk, Phys. Rept. 405 (2005) 279 [hep-ph/0404175].

[3] F. Takayama and M. Yamaguchi, Phys. Lett. B 485 (2000) 388 hep-ph/0005214. 
[4] W. Buchmuller, L. Covi, K. Hamaguchi, A. Ibarra and T. Yanagida, JHEP 0703 (2007) 037 hep-ph/0702184.

[5] For a recent discussion and references, see for example,

M. Cirelli, E. Moulin, P. Panci, P. D. Serpico and A. Viana, arXiv:1205.5283 [astro-ph.CO].

[6] A. Arvanitaki, S. Dimopoulos, S. Dubovsky, P. W. Graham, R. Harnik and S. Rajendran, Phys. Rev. D 79 (2009) 105022 arXiv:0812.2075 [hep-ph]].

[7] W. B. Atwood et al. [LAT Collaboration], Astrophys. J. 697 (2009) 1071 arXiv:0902.1089 [astro-ph.IM]].

[8] A. A. Abdo et al., Phys. Rev. Lett. 104 (2010) 091302 arXiv:1001.4836 [astroph.HE]].

[9] M. Ackermann et al. [LAT Collaboration], arXiv:1205.2739 [astro-ph.HE].

[10] G. Vertongen and C. Weniger, JCAP 1105 (2011) 027 arXiv:1101.2610 [hep-ph]].

[11] T. Bringmann, X. Huang, A. Ibarra, S. Vogl and C. Weniger, arXiv:1203.1312 [hep-ph].

[12] C. Weniger, arXiv:1204.2797 [hep-ph].

[13] S. Profumo and T. Linden, arXiv:1204.6047 [astro-ph.HE].

[14] E. Tempel, A. Hektor and M. Raidal, arXiv:1205.1045 [hep-ph].

[15] A. Boyarsky, D. Malyshev and O. Ruchayskiy, arXiv:1205.4700 [astro-ph.HE].

[16] L. Bergstrom, arXiv:1205.4882 [astro-ph.HE].

[17] A. Ibarra, S. Lopez Gehler and M. Pato, arXiv:1205.0007 [hep-ph]. A. Rajaraman, T. M. P. Tait and D. Whiteson, arXiv:1205.4723 [hep-ph].

[18] E. Dudas, Y. Mambrini, S. Pokorski and A. Romagnoni, arXiv:1205.1520 [hep-ph]. J. M. Cline, arXiv:1205.2688 [hep-ph]. K. -Y. Choi and O. Seto, arXiv:1205.3276 [hep-ph]. H. M. Lee, M. Park and W. -I. Park, arXiv:1205.4675 [hep-ph]. B. Kyae and J. -C. Park, arXiv:1205.4151 [hep-ph]. B. S. Acharya, G. Kane, P. Kumar, R. Lu and B. Zheng, arXiv:1205.5789 [hep-ph]. M. R. Buckley 
and D. Hooper, arXiv:1205.6811 [hep-ph]. X. Chu, T. Hambye, T. Scarna and M. H. G. Tytgat, arXiv:1206.2279 [hep-ph]. D. Das, U. Ellwanger and P. Mitropoulos, arXiv:1206.2639 [hep-ph]. Z. Kang, T. Li, J. Li and Y. Liu, arXiv:1206.2863 [hep-ph]. N. Weiner and I. Yavin, arXiv:1206.2910 [hep-ph]. L. Feng, Q. Yuan and Y. -Z. Fan, arXiv:1206.4758 [astro-ph.HE].

[19] M. Su and D. P. Finkbeiner, arXiv:1206.1616 [astro-ph.HE].

[20] M. Ibe and T. T. Yanagida, Phys. Lett. B 709 (2012) 374 arXiv:1112.2462 [hepph]]; M. Ibe, S. Matsumoto and T. T. Yanagida, Phys. Rev. D 85 (2012) 095011 arXiv:1202.2253 [hep-ph]].

[21] W. Buchmuller, V. Domcke and K. Schmitz, Phys. Lett. B 713 (2012) 63 arXiv:1203.0285 [hep-ph]].

[22] T. Moroi and L. Randall, Nucl. Phys. B 570 (2000) 455 hep-ph/9906527.

[23] R. Kitano, H. Murayama and M. Ratz, Phys. Lett. B 669 (2008) 145 arXiv:0807.4313 [hep-ph]].

[24] B. S. Acharya, G. Kane and P. Kumar, Int. J. Mod. Phys. A 27 (2012) 1230012 arXiv:1204.2795 [hep-ph]].

[25] A. Boyarsky, D. Malyshev and O. Ruchayskiy, Phys. Lett. B 705 (2011) 165 arXiv:1012.5839 [hep-ph]].

[26] M. Ackermann et al. [LAT Collaboration], arXiv:1205.6474 [astro-ph.CO].

[27] M. Ackermann, M. Ajello, A. Allafort, L. Baldini, J. Ballet, G. Barbiellini, D. Bastieri and K. Bechtol et al., JCAP 1005 (2010) 025 arXiv:1002.2239 [astroph.CO]].

[28] J. Ripken, J. Conrad and P. Scott, JCAP 1111 (2011) 004 arXiv:1012.3939 [astroph.HE]].

[29] M. Ackermann et al. [Fermi-LAT Collaboration], Phys. Rev. Lett. 107 (2011) 241302 arXiv:1108.3546 [astro-ph.HE]].

[30] G. Bertone, W. Buchmuller, L. Covi and A. Ibarra, JCAP 0711 (2007) 003 arXiv:0709.2299 [astro-ph]]. 
[31] T. Sjostrand, S. Mrenna and P. Z. Skands, Comput. Phys. Commun. 178 (2008) 852 arXiv:0710.3820.

[32] M. Su, T. R. Slatyer and D. P. Finkbeiner, Astrophys. J. 724 (2010) 1044 arXiv:1005.5480 [astro-ph.HE]].

[33] L. Bergstrom and P. Ullio, Nucl. Phys. B 504 (1997) 27 hep-ph/9706232]; P. Ullio and L. Bergstrom, Phys. Rev. D 57 (1998) 1962 hep-ph/9707333.

[34] M. Cirelli, N. Fornengo and A. Strumia, Nucl. Phys. B 753 (2006) 178 hep$\mathrm{ph} / 0512090]$.

[35] A. Hryczuk and R. Iengo, JHEP 1201 (2012) 163 [arXiv:1111.2916 [hep-ph]].

[36] O. Adriani et al. [PAMELA Collaboration], Phys. Rev. Lett. 105 (2010) 121101 arXiv:1007.0821 [astro-ph.HE]].

[37] A. Ibarra and D. Tran, JCAP 0807 (2008) 002 [arXiv:0804.4596 [astro-ph]].

[38] F. Donato, D. Maurin, P. Brun, T. Delahaye and P. Salati, Phys. Rev. Lett. 102 (2009) 071301 arXiv:0810.5292 [astro-ph]].

[39] W. Buchmuller, A. Ibarra, T. Shindou, F. Takayama and D. Tran, JCAP 0909 (2009) 021 arXiv:0906.1187[hep-ph]].

[40] M. Garny, A. Ibarra and S. Vogl, JCAP 1107 (2011) 028 arXiv:1105.5367 [hep$\mathrm{ph}]$.

[41] C. Evoli, I. Cholis, D. Grasso, L. Maccione and P. Ullio, arXiv:1108.0664 [astroph.HE].

[42] M. Garny, A. Ibarra, and D. Tran, arXiv:1205.6783 [hep-ph].

[43] T. Bringmann and P. Salati, Phys. Rev. D 75 (2007) 083006 astro-ph/0612514].

[44] F. Donato, D. Maurin, P. Salati, A. Barrau, G. Boudoul and R. Taillet, Astrophys. J. 563 (2001) 172 astro-ph/0103150].

[45] T. Bringmann, L. Bergstrom and J. Edsjo, JHEP 0801 (2008) 049 arXiv:0710.3169 [hep-ph]]. 
[46] M. Garny, A. Ibarra, D. Tran and C. Weniger, JCAP 1101 (2011) 032 arXiv:1011.3786 [hep-ph]].

[47] J. F. Navarro, C. S. Frenk, S. D. M. White, Astrophys. J. 462 (1996) 563-575. astro-ph/9508025] J. F. Navarro, C. S. Frenk, S. D. M. White, Astrophys. J. 490 (1997) 493-508. [astro-ph/9611107].

[48] J. F. Navarro, E. Hayashi, C. Power, A. Jenkins, C. S. Frenk, S. D. M. White, V. Springel, J. Stadel et al., Mon. Not. Roy. Astron. Soc. 349 (2004) 1039. astroph/0311231]. A. W. Graham, D. Merritt, B. Moore, J. Diemand, B. Terzic, Astron. J. 132 (2006) 2701-2710. astro-ph/0608613.

[49] F. Iocco, M. Pato, G. Bertone and P. Jetzer, JCAP 1111 (2011) 029 arXiv:1107.5810 [astro-ph.GA]].

[50] V. Springel, J. Wang, M. Vogelsberger, A. Ludlow, A. Jenkins, A. Helmi, J. F. Navarro and C. S. Frenk et al., Mon. Not. Roy. Astron. Soc. 391 (2008) 1685 [arXiv:0809.0898 [astro-ph]].

[51] J. Wess and J. Bagger, Supersymmetry and supergravity, Princeton, USA: Univ. Pr. (1992) 259 p.

[52] T. Moroi, Effects of the gravitino on the inflationary universe, hep-ph/9503210.

[53] S. Bobrovskyi, W. Buchmuller, J. Hajer and J. Schmidt, JHEP 1010 (2010) 061 arXiv:1007.5007 [hep-ph]].

[54] S. Bobrovskyi, J. Hajer and S. Rydbeck, in preparation.

[55] L. Covi, M. Grefe, A. Ibarra and D. Tran, JCAP 0901 (2009) 029 arXiv:0809.5030 [hep-ph]].

[56] M. Grefe, Neutrino signals from gravitino dark matter with broken R-parity, DESYTHESIS-2008-043. 